\title{
A Simulation Environment to Support Prognostics and Health Management in Smart Manufacturing Systems
}

\author{
A Thesis \\ Presented to the Faculty of the School of \\ EngineERING AND Applied ScIEnCE \\ UNIVERSITY OF VIRGINIA
}

Author:

Advisor:

Gerald R. Heddy, III

Dr. Peter Beling

In Partial Fulfillment of the requirements for the Degree

Master of Science in Systems Engineering

June 2016 


\section{Abstract}

Maintenance activities are a significant part of an advanced manufacturer's budget and could potentially represent an organization's source of vulnerability in sustaining a stable supply chain. It is crucial for a manufacturer to understand the implications of maintenance on their organization's ability to produce quality products in an efficient time-frame at an acceptable cost. Furthermore, it can be expensive, time consuming, and create a risk to finished product supply to evaluate results from altering maintenance activities and policies during a production cycle. This is especially true when attempting to implement prognostics and health management (PHM) methods on existing smart manufacturing systems (SMS). This thesis presents research studying the trade-offs of various maintenance policies, including PHM methods, using a simulation with the purpose of providing decision support. The modeling approach involves building the structure for a simulation in which maintenance policies, probabilistic models of failure rates, processing speeds, and costs are inputs into the simulation environment. This generality allows for quickly altering a manufacturing model, and the ability to consider novel policies where control of machine parameters is needed. The outputs of the simulation evaluate policies in terms of cost per unit produced and overall equipment effectiveness (OEE), a widely used industry metric. Data from industrial partners was used in the validation of the simulation environment. This work is part of a larger attempt to equip plant managers and business leaders with the methods for understanding which PHM strategy is best suited for their manufacturing system. 


\section{Acknowledgments}

I would like to thank my advisor, Dr. Peter Beling and his research team for their continued support and guidance through my research and preparation of this thesis work. I am grateful to my committee members, Dr. Yacov Haimes, Dr. Stephen Adams, Dr. James Lambert, and Dr. Cody Fleming for their additional help and guidance. I would like to thank the National Institute of Standards and Technology for providing funding and outstanding technical expertise. My parents and family deserve copious recognition for their unrelenting encouragement and lifelong commitment that has allowed me pursue my aspirations. My better half, Colleen Curley, was instrumental in motivating and mentoring me to complete this research. I am also thankful for my friends, without whom I would most certainly be quite bored and uninteresting.

This thesis is dedicated to my grandparents Edward Kozar, Elizabeth Kozar, Gerald Heddy, Sr., and Anita Heddy, and my mentor Robert 'Bob' Peel. Their influences have all collectively made me into the person I am now and will continue to be. 


\section{Contents}

$\begin{array}{ll}\text { Abstract } & \text { i }\end{array}$

Acknowledgments $\quad$ ii

List of Figures $\quad$ vi

1 Introduction 1

1.1 Motivation .......................... 1

1.2 Purpose and Scope . . . . . . . . . . . . . . . . . . . 4

1.3 Organization of the Thesis . . . . . . . . . . . . . 7

2 Background $\quad 8$

2.1 Chapter Overview . . . . . . . . . . . . . . . . 8

2.2 Maintenance Policies in Smart Manufacturing Systems . . . . . . . . 9

2.2.1 Corrective Maintenance . . . . . . . . . . . . . . . . . . 10

2.2.2 Time Based Maintenance . . . . . . . . . . . . . . . . . . 12

2.2.3 Condition Based Maintenance . . . . . . . . . . . . . . . 13

2.2.4 Extending CBM Policies . . . . . . . . . . . . . . . 15

2.3 Simulation of Maintenance in Manufacturing . . . . . . . . . . . . 17

2.4 Chapter Summary . . . . . . . . . . . . . . . . . . . . . 21

3 Methodological Approach $\quad 22$

3.1 Chapter Overview . . . . . . . . . . . . . . . . . . . . 22

3.2 Simulation Methodology Supporting PHM in SMS . . . . . . . . . . 24

3.2.1 Formulation .................... 25 
3.2.2 Building, Testing, and Production Runs . . . . . . . . . . . . 29

3.2.3 Metrics, Analysis, and Trade-offs . . . . . . . . . . . . 32

3.3 Simulation Design . . . . . . . . . . . . . . . . . 37

3.4 Chapter Summary . . . . . . . . . . . . . . . . . . 45

4 Demonstration of Method 46

4.1 Chapter Overview . . . . . . . . . . . . . . . . 46

4.2 Advanced Manufacturer Implementation . . . . . . . . . . . . . 46

4.3 Discussion of Results . . . . . . . . . . . . . . . . . . 53

4.4 Chapter Summary . . . . . . . . . . . . . . . . . . . 59

5 Conclusions $\quad 60$

5.1 Chapter Overview . . . . . . . . . . . . . . . 60

5.2 Findings and Contributions . . . . . . . . . . . . 60

5.3 Future Work . . . . . . . . . . . . . . . . . 62

5.4 Chapter Summary . . . . . . . . . . . . . . . . 64

Appendix A: MATLAB Interface Subcomponents $\quad 71$ 


\section{List of Figures}

- Figure 1, Page 6. Implementing a PHM program in a SMS system

- Figure 2, Page 11. Visual representation of maintenance policies found in the literature

- Figure 3, Page 18. Representation of a general simulation methodology and the steps taken

- Figure 4, Page 19. A literature review table of attributes that illustrates existing work in SMS maintenance simulation

- Figure 5, Page 23. The PHM specific simulation methodology for a SMS showing the steps taken by a systems analyst to build a business case

- Figure 6, Page 30. A view of the simulation structure showing inputs, system, and outputs

- Figure 7, Page 48. Tool Company Production Line Hierarchy

- Figure 8, Page 51. Tool Company Lathe processing times fit to a Weibull distribution

- Figure 9, Page 52. Simulation built in MATLAB for a Tool Company implementation

- Figure 10, Page 54. Results showing that cost per unit decreases and OEE rises as the Tool Company drives towards implementing a CBM policy 
- Figure 11, Page 55. Results comparing the cost to implement each policy versus OEE

- Figure 12, Page 57. Unit cost versus policy cost to implement

- Figure 13, Page 58. Maintenance labor hours were totaled and converted to show the number of employees needed for each policy as a CDF

- Figure 14, Page 63. Simulation structure suggesting the direction of future work by interfacing with a decision module

- Figure 15, Page 72. View of the cutoff saw process

- Figure 16, Page 73. View of the process for a component of the cutoff saw, the saw blade

- Figure 17, Page 74. View of the assembly process, which has two servers for the laser and inspection processes, respectively 


\section{Introduction}

\subsection{Motivation}

Industries active in the manufacturing sector exist in a competitive landscape where profitability is heavily influenced by their operational directives. Capital intensive businesses, for example the automotive and airline industries, are under intense pressure to keep their production costs low and guarantee high utilization of assets. A crucial process that operates at the intersection of sustaining high asset utilization and low costs is the maintenance policy a manufacturer has selected to follow. If not chosen or managed wisely, maintenance policies can have adverse effects on production costs, product quality, people safety, and even employee morale. For benchmarking, decision makers should have information that reflects a maintenance policy's impact on standard metrics such as (1) cost per unit produced, (2) cost of policy implementation, (3) overall equipment effectiveness (OEE), and (4) labor hours needed for 
maintenance.

Fortunately, the rise of Smart Manufacturing Systems (SMS) has the potential to change the way organizations make operations decisions so that they may drive down costs, improve product quality, benefit employee safety, and ultimately propagate efficiencies throughout the enterprise. A SMS brings the ability to leverage hierarchical information systems and real-time data analytics to implement immediate control strategies over the systems. The SMS does this by employing a broad application of networked information-based technologies throughout a company's supply chain [1]. SMS sit adjacent to the Internet of Things (IoT) and Big Data revolutions whereby connected machines can be monitored and controlled from anywhere, and an abundance of information is available to make data-driven decisions. While there is not a shortage of technologies available to enable smart manufacturing, there exists a lack of modern methods with which to establish and utilize them. Evidence of this can be seen in a recent report on the current state of manufacturing in the United States which called for the need to fill the gap by developing new methodologies. [2]

To fill the void, the field of Prognostics and Health Management (PHM) attempts to create the systems and methods which manufacturers employ to enhance their asset maintenance programs. PHM policies are implemented as a better alternative to traditional corrective maintenance programs primarily defined by initiating action only after a breakdown or some lost production time event has occurred. It is through the use of condition-monitoring, diagnostic, and prognostic methods that PHM attempts to understand the health states of the system and create a manufacturing environment where maintenance is carried out on a preventive, predictive, and proactive basis. More specifically, PHM approaches maintenance by utilizing signals, 
measurements, models, and algorithms to detect, assess, and track degraded health to predict failure progression [3]. A PHM maintenance policy proves beneficial by reducing manufacturer dependence on non-value added maintenance time and capital of parts replacement. PHM strives to increase asset lifespan while operating at lower cost.

However, even after a method is selected or created, a distinct challenge to the adoption of PHM methods in smart manufacturing systems is estimating their impact on the system and its subsystems. This is owed to the nature of a modern, competitive manufacturing system characterized by the complex composition of equipment, processes, and people. It often proves too difficult to measure the financial impacts and develop a business case by experimenting with the actual system, building a physical model, or deriving an analytical solution. Thus, simulation lends itself as the tool of choice and has been widely used when evaluating complex manufacturing environments for the comparison of maintenance policy decisions. Simulation allows for the modeling of complex manufacturing systems with stochastic elements that cannot be evaluated analytically, can maintain better control over the system than would be possible if the manufacturer tested on its actual production lines, can compare alternative maintenance strategies in a short time-frame without impacting the current manufacturing system, and can study a large period of time in a short interval $[4]$. 


\subsection{Purpose and Scope}

The aim of this thesis is to present a simulation methodology that builds the business case for supporting prognostics and health management (PHM) in smart manufacturing systems (SMS). As compared to past efforts, the methodology targets the ability to (1) simulate novel PHM policies, (2) adds generality for the purpose of quickly updating a manufacturing model, and (3) provides the ability to integrate with a decision module for the purpose of determining optimal policies. These additions can be achieved by structuring the simulation such that maintenance policies, stochastic models of failures, processing speeds, costs, mean time to repair equipment, etc. are inputs into the simulation environment. Trade-offs such as cost per unit, cost of policy, OEE, and maintenance labor hours required are structured as outputs from the simulation. It will also consider the manufacturing system hierarchically, allowing the decision-maker to detail input parameters and collect output data down to the equipment component level.

Previous work on building business cases for implementing PHM methods in SMS using simulation has targeted specific applications such as electronic systems [5] and helicopter avionics [6]. When it comes to developing the business case for implementing PHM in smart manufacturing systems, the literature could benefit from a formal presentation of a simulation method with a general input-output structure capable of considering new maintenance policies employing advanced PHM techniques such as considering machine parameters as decision variables. Traditional reliability literature does provide simulation models for comparing maintenance policies [7],

but can be augmented by reconciling their models with PHM techniques, modeling hierarchically, and considering both cost and OEE metrics for analysis. 
This thesis focuses on the advanced manufacturing setting, and will be most helpful when applying the method to those industries which operate in complex environments using smart manufacturing technologies. The simulation method will be demonstrated with input data from an advanced manufacturer employing SMS technologies and investigating where their factory could benefit from PHM. The resulting simulation, built in MATLAB, will be used to compare several maintenance policies found in the literature for their cost and equipment effectiveness. This includes considering a Corrective Maintenance policy, whereby maintenance activities are only performed at the time of system failure, and preventive policies that attempt to plan for failures by performing maintenance before they occur. Preventive maintenance is the topic of an exhaustive amount of literature and extends to many subtopics including time based maintenance (TBM) and condition based maintenance (CBM). CBM also reaches further to include an array of policies, many of which are the topic of current literature.

Figure 1 illustrates how this thesis fits into the larger effort to equip manufacturing leaders and systems analysts with the methods for understanding how to achieve a PHM implementation in an existing system. The simulation methodology is the second stage of implementation and an essential piece intended to complement the broader body of work representing a holistic vision for enabling manufacturer's to adopt PHM techniques. 


\section{Implementing a PHM Program in SMS}

- Identify Targets for a PHM System via Risk Methods

Scope

- Simulation Environment to Support Business Case Justification of PHM in SMS

.

- Decision Support with PHM Information (AM-PHM)

Figure 1: For a PHM implementation, a manufacturer would follow along the three stages represented in this figure. This thesis is primarily concerned with the Simulation stage and providing decision-makers with informed trade-off analysis based on a PHM focused simulation methodology. 


\subsection{Organization of the Thesis}

This thesis is arranged to include a literature review in Chapter 2 focused on the current state of maintenance policies and the use of simulation in manufacturing. Chapter 3 will review the simulation methodology. In Chapter 4, an application of the simulation method will be presented using data from a advanced manufacturer. Chapter 5 will conclude the thesis by discussing findings and considerations, contributions, and potential for future work. The appendices will show detailed simulation data and the MATLAB SimEvents workspace. 


\section{Background}

\subsection{Chapter Overview}

This chapter reviews topics presented in the literature and lays out the theoretical basis of the thesis. It details the current state of maintenance in manufacturing and how the study of maintenance policies has evolved. A map of how the policies are related can be seen in Figure 2. The use of simulation in manufacturing is discussed with a focus on simulation environments constructed specifically for manufacturing maintenance. The chapter exposes gaps in the literature and closes by suggesting that a holistic simulation method for driving PHM decision support with industry substantiated metrics is needed to augment existing approaches. 


\subsection{Maintenance Policies in Smart Manufacturing Systems}

Maintenance is defined among the literature as a set of activities or tasks used to restore an asset to a state in which it can perform its designed functions [8]. Maintenance policies can be broadly classified into Corrective Maintenance, sometimes called reactive, and Preventive Maintenance policies [9]. Preventive maintenance (PM) policies in particular have been the topic of research and development for a long time. The field of operations research introduced the application of PM policies based on mathematical descriptions as early as the 1950's. An example of this work can be seen in papers by [10] and [11] where they proposed the creation of two optimal preventive maintenance policies and compared policies for stochastically failing equipment, respectively.

Preventive maintenance evolved to be divided into two major categories, Time Based Maintenance (TBM) and Condition Based Maintenance (CBM). CBM is the parent discipline of most recent literature and therefore contains the broader spectrum of policy development as compared to TBM. The domain of Prognostics and Health Management (PHM) is mostly contained in and concerned with CBM practices. PHM technologies reduce time and costs for maintenance of products or processes through efficient and cost-effective diagnostic and prognostic activities. Diagnostics is defined as process of finding the source of faults [12]. This is valuable to a manufacturer since the aim of diagnosis is to provide early warning signs that equipment is about to fail; the obvious and critical assumption being that there remains a period of time whereby maintenance activities can still be performed before eventual failure. In order to address this timing concern, prognosis must be practiced. Prognostic methods endeavor to provide a prediction of when a failure will occur at a time 
interval greater than a diagnosis can provide.

It is not uncommon for PHM, and other forms of maintenance, to be discussed from either a product or process point of view. Product PHM, which is more common, refers to health monitoring, diagnostics, or prognostics of a finished system such as a car or aircraft. Process PHM is for a system that incorporates one or multiple pieces of equipment to complete a task such as an assembly or machining process [13] [14] [15] [16]. Processes in manufacturing will be the focus of this thesis, and the policies reviewed will be at both the component and higher system levels. It is also important to note that this work is primarily concerned with PHM implementations for existing manufacturing processes. While a new production facility could most certainly use the simulation method to build a business case, the data for validation of current processes will not be available, and thus possibly difficult to estimate. The use of interviewing subject matter experts and benchmarking other similar existing processes would have to be used. Implementation of PHM techniques on existing processes is often the more interesting and challenging case, since the cost to retool equipment and retrain or hire employees may be significant.

Figure 2 illustrates a general overview of the literature and the relationship of how maintenance policies can be categorized.

\subsubsection{Corrective Maintenance}

Corrective maintenance $(\mathrm{CM})$, often referred to as reactive maintenance, is the easiest policy to understand and for a manufacturer to implement. Under CM, the manufacturing system would only perform maintenance activities after a failure has occurred. There are a few variations in the literature as to the exact policy being carried out, 


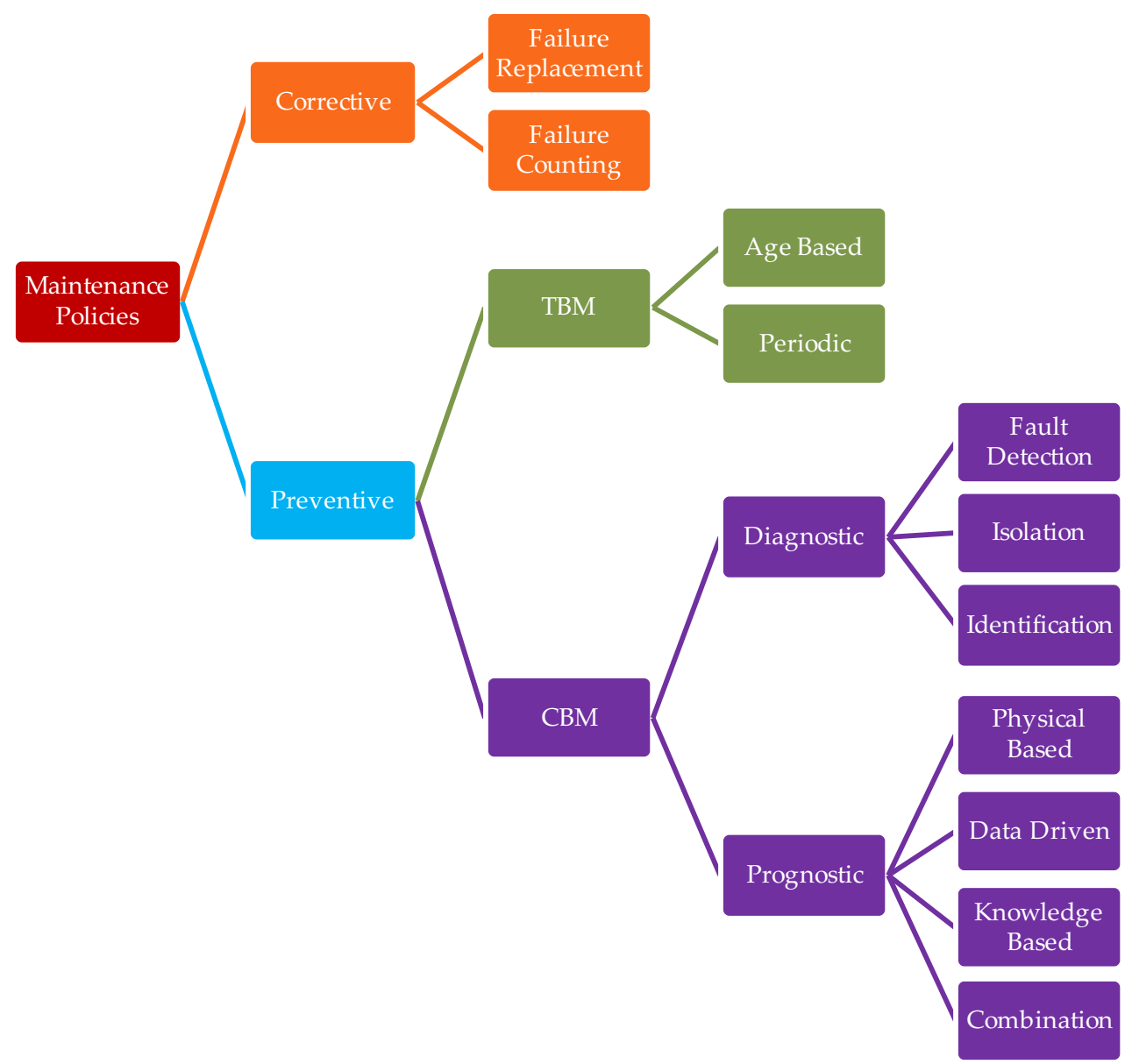

Figure 2: This is a visual representation of maintenance policies found in the literature. It should be noted that it is not an exhaustive list, and that most industry maintenance policies are implemented as a hybrid of several policies. 
dependent upon the system. For example, replacement of a component could happen immediately after a failure or after a specified number of failures [17]. Reactive maintenance could be the best solution in situations where failures have little to near zero impact on cost and system uptime, especially as compared to the cost of implementing more progressive maintenance strategies. For example, light bulbs might be something that are only changed after failure. Equipment and their respective components that are best suited for CM would most likely be filtered out during the first stage of a PHM implementation, as represented in Figure 1.

\subsubsection{Time Based Maintenance}

Time Based Maintenance (TBM) is often a manufacturer's first foray into the realm of preventive maintenance. This is because it can be simpler to implement since maintenance activities are performed based upon a specific unit of time $T$ or period: meaning only a schedule, clock, or cycle counter is needed from an equipment perspective. There are several policies proposed in the literature that qualify as TBM. For example, in a survey by [17] age-dependent, periodic, and sequential could all be categories of TBM policies. In an age based policy, maintenance activities are performed when a piece of equipment or component reaches a predetermined time $T$ or fails. Periodic denotes a policy where maintenance is performed at time intervals $k T$ where $k=(0,1,2,3, \ldots)$, regardless of failures that previously occurred. Sequential means that the time interval $T$ for performing maintenance is changing, usually decreasing, as the component ages.

TBM policies generally assume a stochastic nature for component mean time between failure (MTBF), and that the system is deteriorating with the passage of 
time [18]. The challenge to manufacturer's is to correctly estimate the randomness of their equipment's MTBF. This has been posed with the help of fitting probability distributions such as the normal, the gamma, or Weibull [11]. The Weibull will be the distribution used in Chapter 4 when fitting data from an advanced manufacturer for generating failures. A major limitation to TBM may be the increased cost due to performing more maintenance activities than actually required. This can occur is the time interval $T$ is too short. Conversely, a $T$ set too aggressively close to the mean failure time will allow for deviations to occur resulting in frequent corrective maintenance activities, especially for equipment with large failure variances.

\subsubsection{Condition Based Maintenance}

Condition Based Maintenance is a policy that aids a manufacturer's maintenance decisions through real-time diagnostics of imminent failures and prognostics of future equipment remaining usable life (RUL), or the health state. The decision to perform maintenance is reached by observing a condition of the system and its components [19]. CBM, sometimes termed predictive maintenance, may ultimately be more cost effective if a process's or equipment's health data accurately reflects its current state and allows a machine to run longer until maintenance, as compared to a TBM policy. CBM is wheelhouse of PHM methods and the topic of most current literature as it represents the intersection of rapidly developing information systems technologies and data analytics techniques.

CBM was found to be more realistic from a conceptual point of view when compared with TBM [20]. This is aided by the findings of [21] where $99 \%$ of equipment

showed a sign or indication that a failure was about to occur. The challenge of 
CBM is gathering sufficient data to make a reasonably accurate prediction. If reliable data is available, CBM was found to be most cost effective when employing a grouping strategy that reacts to changing component deterioration and dependencies in a multi-component system [22].

A general formula for the manufacturing cost of a CBM type policy as given by [23] and used in calculation for the simulation method presented in Chapter 3 can be written as:

$$
C_{\text {Total }}=\left(N_{u} * C_{u}\right)+\left(N_{m} * C_{m}\right)+C_{d} \int_{0}^{T}(1-A(t)) d t
$$

Where $T$ is the lifetime of the system under review and,

$$
\begin{aligned}
N_{u} & =\text { number of unplanned failures up to time } T \\
C_{u} & =\text { cost of an unplanned failure } \\
N_{m} & =\text { number of PM actions completed within time } T \\
C_{m} & =\text { cost of a PM activity } \\
C_{d} & =\text { cost rate of downtime } \\
A(t) & =\text { Availability, or percentage of time in operational state }
\end{aligned}
$$

In 2010, a comprehensive review was conducted of prognostic and diagnostic methodologies for CBM that presented existing policies in four categories: physi- 
cal models, knowledge-based models, data-driven models, and combination (hybrid) models [19]. This review highlighted the successes and limitations of many specific methods across these four categories such as Hidden Markov Models, Bayesian network-related methods, Fuzzy Logic, and Principal Components Analysis. No one method stood out as being sufficient to provide both diagnostic and prognostic intelligence at multiple levels. This review demonstrated that for every method's strength, there was at least a single weakness.

\subsubsection{Extending CBM Policies}

An exciting contribution of this thesis is the ability for the simulation method to consider novel maintenance policies seeking to extend CBM approaches. Adaptive Multi-Scale PHM (AM-PHM) is an example of one such policy [24]. AM-PHM fits in the maintenance policy map (defined visually in Figure 2) under Condition Based Maintenance as a hybrid of diagnostic and prognostic policies employing knowledgebased and data-driven techniques. It was first posed to address the existing gap in

providing PHM for hierarchical manufacturing systems, which exist abundantly in large-scale corporations.

AM-PHM incorporates multi-level, hierarchical relationships and PHM information gathered from a manufacturing system. Diagnostic and prognostic information regarding the current health of the system and its collective components are utilized and propagated up the hierarchical structure. The goal is to create actionable prognostic and diagnostic intelligence along the manufacturing process hierarchy. This information is assumed to include the predicted health state upon completion of a task. Thus, the benefit of an AM-PHM policy is more holistic, system-wide decision- 
making to increase cost efficiency and equipment effectiveness with respective to business goals.

New CBM policies, such as AM-PHM, will reject the notion that equipment parameters must only be optimized with respect to their specific manufacturing process. For example, in a typical machining process, a lathe is normally setup with parameters to achieve maximum efficiency and a minimum smoothness specification. However, in complex systems of systems this optimized element represents a trade-off of other elements. Each component of a manufacturing system is available with a multidimensional spectrum of available parameter settings, not merely a one-dimensional set of values. This type of policy can leverage hierarchical information and the ability to manipulate system parameters to realize cost and equipment effectiveness gains that would ordinarily be missed with a less holistic policy.

No policy, however, is without its drawbacks. AM-PHM and others like it would certainly require a high cost to implement on an existing manufacturing process that did not already collect high-quality sources of diagnostic and prognostic data. A company without the capital to invest in Enterprise Resource Planning (ERP) systems that could propagate information up the hierarchy would also be less inclined to pursue this type of advanced decision support method. 


\subsection{Simulation of Maintenance in Manufacturing}

Simulation has been used in past and present literature to assess the impact of a maintenance policy on a manufacturing system. Manufacturing generally is the topic of much simulation efforts in practice, and could arguably be the arena of more simulation than any other application area. This is due to the fact that a localized change can be tracked and understood system-wide; and manufacturing systems tend to be complex enough that small changes do not give intuitive understanding of system impact. Figure 3 details the steps taken in a general simulation methodology as given by [4]. These steps will be altered in the next chapter to create a simulation method for analyzing PHM implementations in SMS.

A review of individual simulation studies for maintenance in manufacturing shows the abundance and diverse set of analyses performed. The work of [25] considered and analyzed the effect of five different maintenance policies: corrective maintenance, 30 day preventive, 90 day preventive, event-triggered preventive, and 30 day eventtriggered preventive. This study was to show that Flexible Manufacturing Systems experience big variances for the number of tasks performed depending on the maintenance policy used.

[26] performed a simulation to compare the effect of several maintenance policies on a heterogeneous job shop, where all the machines are performing different tasks. There it was found that adding redundancy of machines does not generally increase throughput, but rather effective preventive maintenance polices can significantly counteract unplanned failures.

Another paper [23] attempted to predict the performance of CBM policies. It accomplished that by using simulation to explore several CBM policies under different 


\section{General Simulation Methodology}

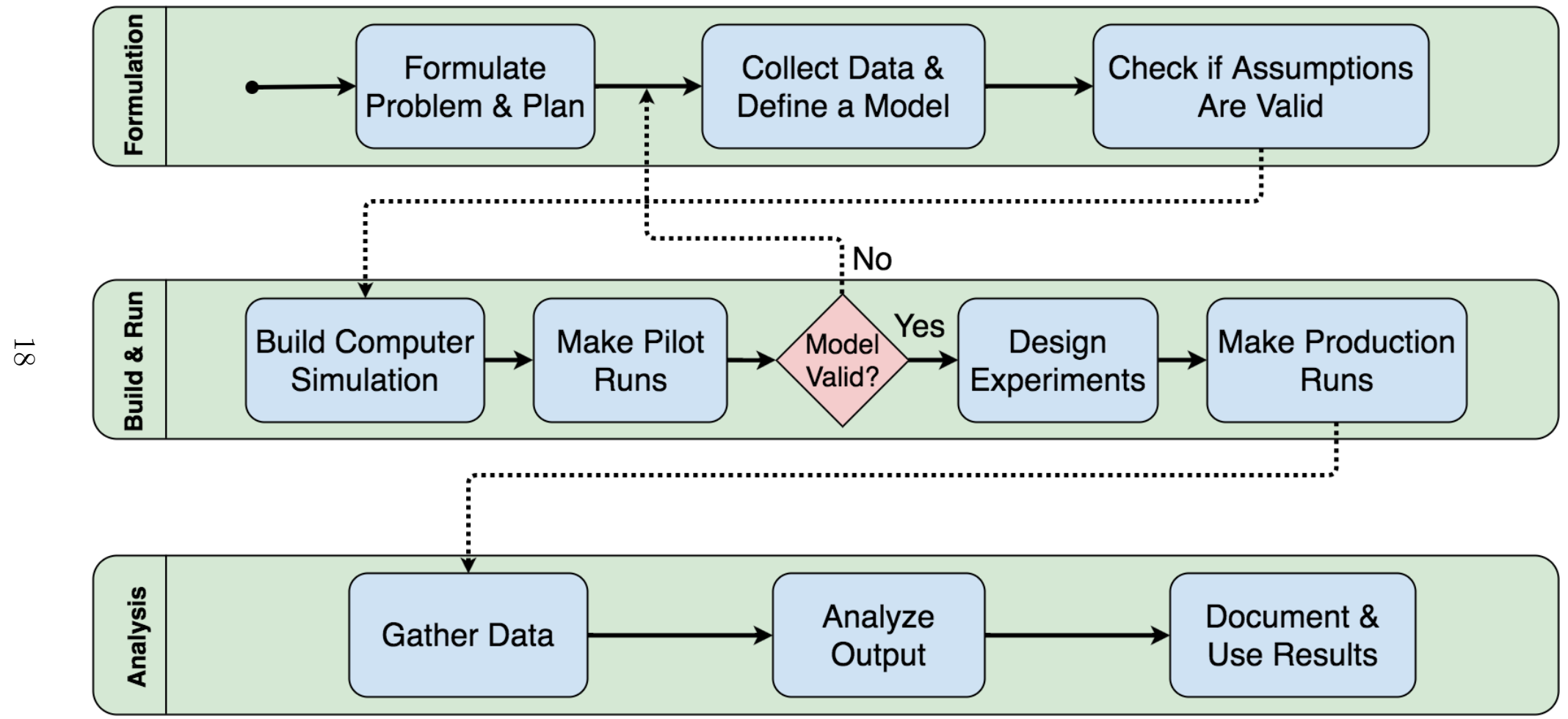

Figure 3: This is a visual representation of a general simulation methodology and the steps taken as found in widely accepted simulation literature [4]. 
Table 1: Attribute table for existing work in SMS maintenance simulation

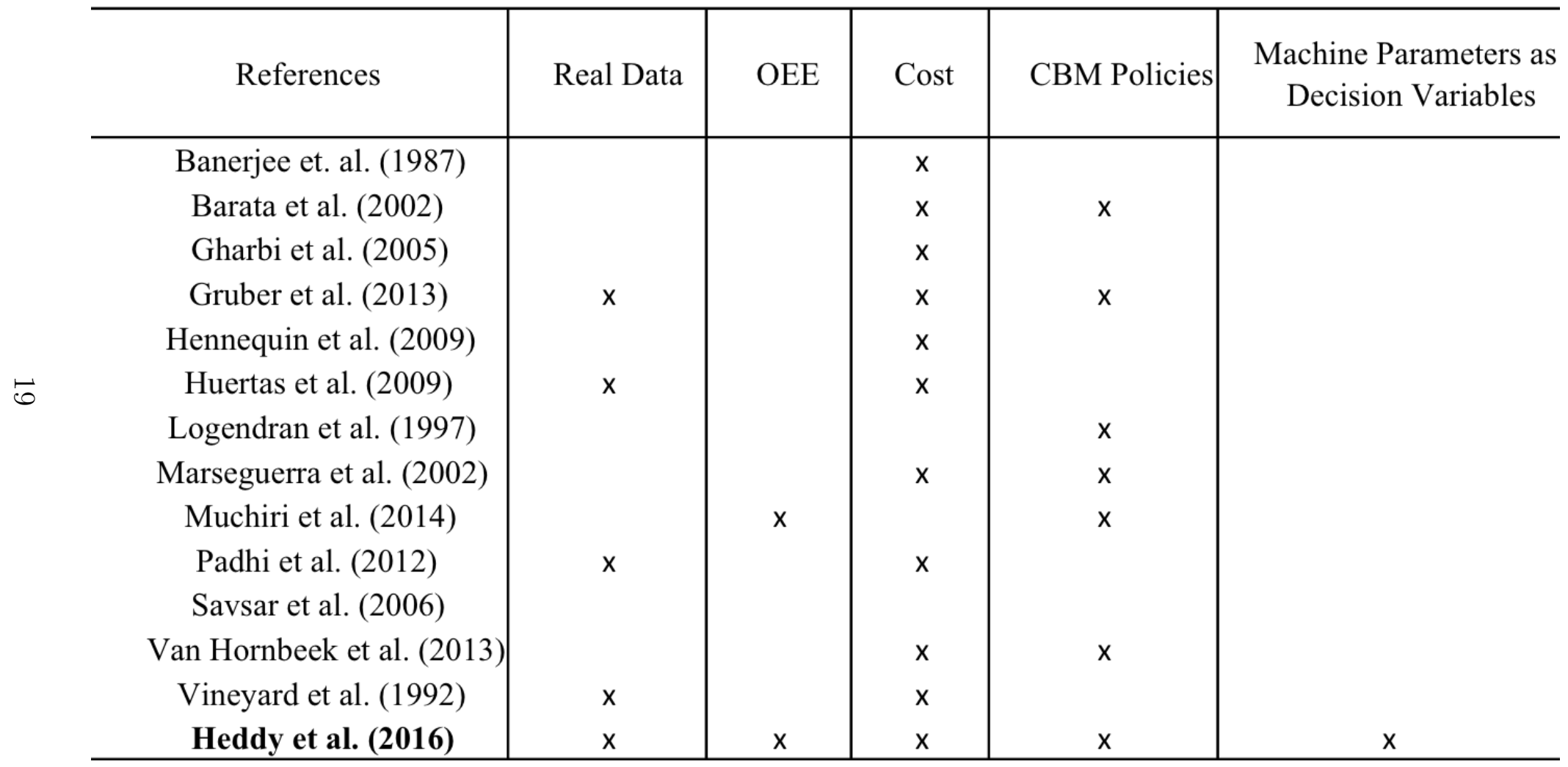

Figure 4: A literature review table of attributes that illustrates existing work in SMS maintenance simulation 
scenarios, and producing a Bayesian network learned from the simulation data to obtain a model for failure prediction.

In yet another paper [27] a simulation model was used to compare the performance of cellular and functional work cell layouts and the effect of maintenance policies. The mean work-in-process (WIP) inventory and throughput times were used to compare the performance of a corrective maintenance and PM policy.

Where there is a significant gap in the literature is a common agreement on which metrics are best suited to compare different maintenance policies. This thesis will build commonly used manufacturing metrics into the simulation structure as outputs. Cost per unit produced, cost of maintenance policy implementation, overall equipment effectiveness (OEE), and the number of hours needed for maintenance activities will all be considered standard outputs. These metrics were found to be valuable to manufacturers through industry visits where the research team sought expert opinions.

The literature also shows a lack of consistency in the method by which manufacturing systems were simulated for the comparison of maintenance policies. This might owe to the fact that there is great diversity of systems, where each is tailored to the product being produced. Simulations are by nature very specific programs built to model unique systems as accurately as possible to answer a systems analysts' questions. This thesis seeks to contribute a simulation method and structure that would allow for easily updated inputs which has the potential to reduce time spent making production runs in PHM implementation analysis. There are methods in existence which use an input-output simulation structure, but most are only built for comparing current operations, excluding maintenance decision support [28] [29]. If 
manufacturing maintenance is analyzed, such as in [7], there is lack of a defined simulation method and consideration of PHM specific attributes like cost and the system hierarchy. A table of attributes that illustrates existing work in SMS maintenance simulation is shown in Figure 4. This table shows how the simulation methodology presented in this thesis compares to current literature and augments prior papers by including all attributes for a more holistic approach.

\subsection{Chapter Summary}

This chapter dissected literature to define various types of maintenance policies and map their relationships. This will be useful information when the simulation method is applied to a real advanced manufacturer environment in chapter 4 . It showed that while there is an abundance of current research to develop condition based maintenance approaches in the field of PHM, there is a lack of unity for a method of simulation to build the respective business case. The next chapter will discuss the method used to close the gap found in the literature. 


\section{Methodological Approach}

\subsection{Chapter Overview}

This chapter reviews the PHM simulation methodology for smart manufacturing systems: formulation, building, testing, making production runs, outputting metrics, performing analysis, and presenting trade-offs to the decision maker. Figure 5 illustrates the steps of the simulation method. Each step is explained in detail, however, the chapter begins with the scoping stage of a PHM implementation and concluding with the exploitation stage as referenced in Figure 1. This chapter concludes with the insight needed to continue with a demonstration of the method where it will be applied it an advanced manufacturer using data captured from an actual production facility. 


\section{PHM Simulation Methodology for SMS}

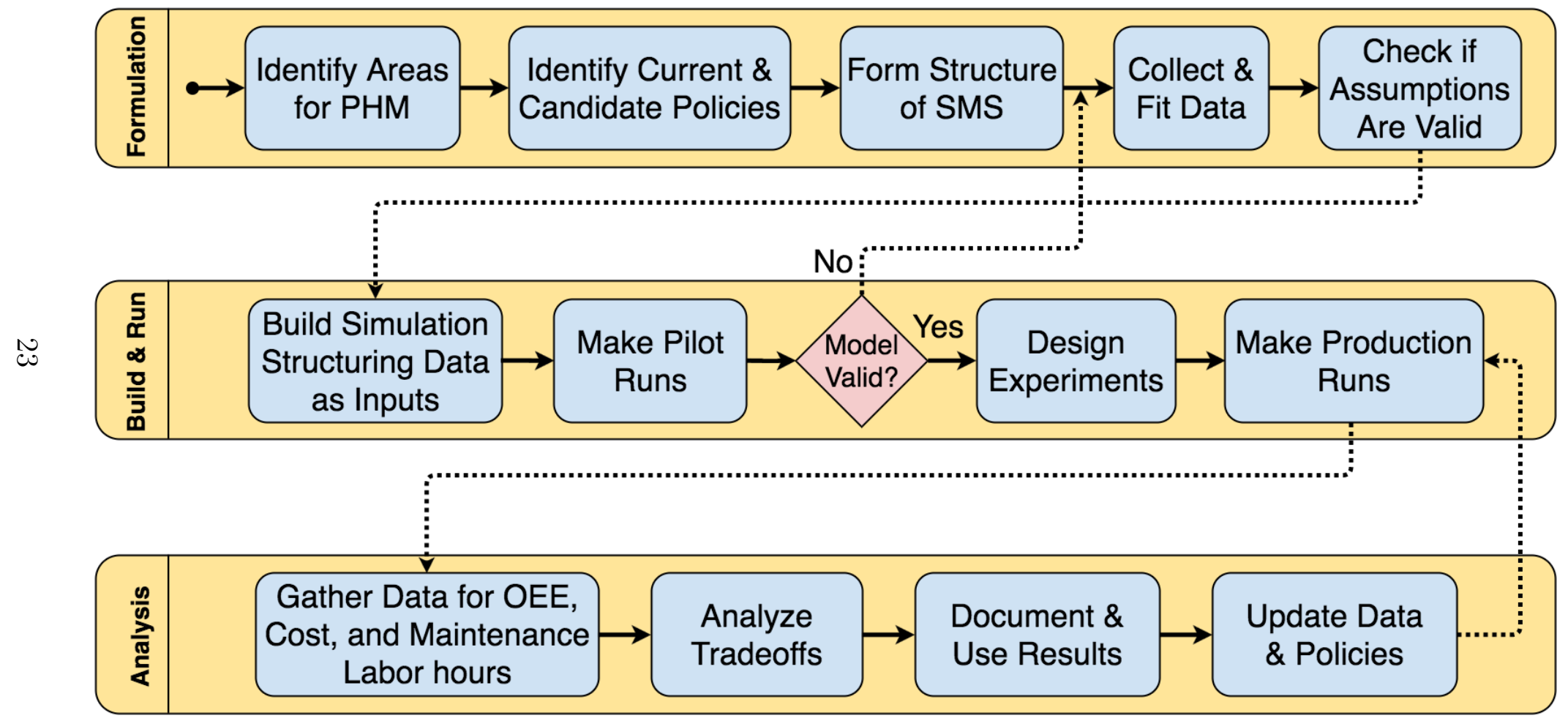

Figure 5: This is a visual representation of the PHM specific simulation methodology for a SMS showing the steps taken by a systems analyst to build a business case. 


\subsection{Simulation Methodology Supporting PHM in SMS}

Building from the general simulation methodology shown in figure 3, this thesis presents a new method depicted in figure 5. The following sections are broken down into (1) formulation, (2) building, testing and production runs, and (3) metrics, analysis, and trade-offs. Each section will explain in detail each step of the simulation methodology. The steps are as follows:

1. Identify Areas for PHM

2. Identify Current and Candidate Policies

3. Form Structure of the SMS

4. Collect and Fit Data

5. Check if Assumptions are Valid

6. Build Simulation Structuring Data as Inputs

7. Make Pilot Runs and Check if the Model is Valid

8. Design Experiments

9. Make Production Runs

10. Gather, Data for OEE, Cost, and Maintenance Labor Hours

11. Analyze Trade-offs

12. Document and Use Results

13. Update Policies and Data, return to step 9 


\subsubsection{Formulation}

PHM implementations have the potential to be quite costly when you add up the costs for sensor installation and activation, research and computing instrumentation for predictive analytics capabilities, and continuous labor for monitoring and managing. If a manufacturer were to blindly introduce a new PHM technique across their entire manufacturing system, there would most likely be pieces of the system which would be over-monitored or micro-managed and not necessarily worth the added expense. Thus, it is important to prioritize exactly where PHM is implemented to guarantee

cost and equipment effectiveness. A system-wide implementation has the potential to stretch corporate budgets and stall PHM implementation. A focused PHM strategy will allow for quicker return on investment, ultimately saving capital which could be reinvested to new cost-saving projects.

This is where the field of Risk Analysis can provide help for identifying prioritized targets in a manufacturing system that most need PHM. Using the combined methods of Hierarchical Holographic Modeling (HHM), Risk Filtering and Ranking Method (RFRM), and Fault Tree Analysis (FTA) the method can achieve step one of the PHM-specific simulation methodology [30], [31], [32]. Work has already been completed on this effort as apart of the aforementioned holistic vision for PHM im-

plementation defined in Figure 1 and presented in this paper [33]. The methods of Risk Analysis (HHM, RFRM, and FCA) were harmonized with the field of PHM and included the creation of PHM-specific rules for determining PHM targets. This thesis's PHM simulation methodology is benefited from applying the risk methods of scoping by streamlining the simulation build process.

The second step in the PHM simulation methodology is to choose the set of can- 
didate maintenance and operations policies to be evaluated. This would be unique to each manufacturer and also entails developing a concrete understanding of which policy is currently being used. It is important to define the current policy being used now for experimental design and trade-off analysis to be performed later. The maintenance policy map shown in Figure 2 can be used a reference for selecting an evaluation set. The demonstration in chapter 4 used candidate policies which represented corrective maintenance, time-based maintenance, and condition-based maintenance. The policies should be chosen seeking to analyze impact on cost reduction and overall equipment effectiveness (OEE) improvements using PHM information for the areas identified in step one. In addition to the current policy being used by the manufacturer, during this step would be the time to note any unused or under utilized PHM technologies. For example, a piece of equipment might have sensing capabilities that are either not activated or being analyzed. This capability will be a factor when developing the set of potential policies, and could bring down the overall cost of implementing a policy.

Each maintenance policy inputed into the simulation for comparison will also have important assumptions and modeling approaches used in reliability theory such as grouping of maintenance activities and the degree of maintenance efficiency. These assumptions should be defined as apart of step two. Grouping is an approach where multiple components are repaired or replaced at the same time to limit aggregate production downtime [34]. Maintenance efficiency speaks to the concept that the activities performed do not return equipment $100 \%$ back to the base condition [35]. Therefore, the systems analyst needs to assume the state of the equipment is either as good as new (AGAN), as bad as old (ABAO), or somewhere in between. The later 
state is usually referred to as imperfect maintenance [36]. The simulation structure, discussed in step 6, is setup such that these maintenance policies and their respective parameters are inputs. The reason for this structure is discussed later as an important contribution.

The third step is to form the structure of the smart manufacturing system. This structure should be hierarchical in nature to ensure capturing the necessary complexity of the manufacturing system as simply as possible. To do this, evaluate and write down or graph the factory hierarchy, but only for the resolution necessary to evaluate cost and OEE impact. Step one and two will inform the decisions made on the level of detail required both up and down the system hierarchy. For example, the demonstration in chapter 4 did not consider every production line in the manufacturing facility, and therefore the production-line was the highest level. Certain equipment components filtered out in step one were also omitted the lowest levels. The system structure might need to be re-evaluated and adjusted in the future as the manufacturing system changes or radically new policies sought to be tested. Any assumptions made in this step should be saved for a validation discussion late. An example of this might be how raw materials and WIP are handled in the factory.

The fourth step is to collect and fit data from the existing manufacturing system. This could prove to be a challenging step if purely corrective maintenance is currently being performed. The manufacturer's systems databases should be carefully analyzed for any helpful information on previous operations history. The data to be collected for input to the simulation could include, but not limited to:

- Processing times for each piece of equipment in the manufacturing process

- Mean Time Between Failure (MTBF) 
- Mean Time To Repair (MTTR)

- Operating costs such as labor, overhead, materials, etc.

- Maintenance costs such as labor, component replacement costs, etc.

- Equipment operating parameters

- Order schedule and SKU characteristics

- Policy implementation costs

The systems analyst will need to be diligent to search for clean data that is backed up by observation. Interviews with experts should be conducted to bolster data collection activities. It is most useful to create a survey form whereby all expert interviews are conducted in a standard manner for a clearer picture of complex manufacturing systems. Data such as MTBF should then be fitted to a distribution; this is easily done by many software packages such as MATLAB or R. Chapter 4 will most often use the Weibull distribution for processing, failure, and repair times. The Weibull is the most widely used distribution in reliability research [7]. Its probability density function is represented as:

$f(x ; \lambda, k)=\frac{k}{\lambda}\left(\frac{x}{\lambda}\right)^{k-1} e^{-(x / \lambda)^{k}}$ for $x \geq 0$

where $k>0$ is the shape parameter and

where $\lambda>0$ is the scale parameter 
The fifth and final step of the formulation stage is to detail and validate the PHM simulation model assumptions. This will require presenting assumptions to the project team and ensuring that the simulation analysis will be able to achieve a comparison of system costs and OEE for each policy selected. Assumptions might have to be made on how equipment deteriorates over time down to the component level, whether the preventive policies considered support a grouping technique to component replacement, the effectiveness of maintenance activities on restoring equipment back to its base-condition, and many other topics covered in the literature.

\subsubsection{Building, Testing, and Production Runs}

The sixth step of the PHM simulation methodology is to build the simulation and to do so with the structure represented in Figure 6. This is an important contribution of this thesis and is the driver behind three main benefits: the ability to simulate new, novel PHM policies, a generality for the purpose of quickly updating the manufacturing model, and capability to integrate with a decision module for the purpose of determining optimal policies. The type of simulation model used in this methodology will be a Discrete Event Simulation (DES) where the system evolves through instantaneous state variable changes at separate points in time. From a PHM perspective, health state variables are added and tracked to infer health state information. When comparing condition-based policies, the simulation will then be able to take advantage of health information before deciding on maintenance activities.

The inputs are anything that can affect how the manufacturing system will operate and additional parameters related to calculating output metrics. The structure clearly

identifies but is not limited to: distribution of processing times for each piece of 


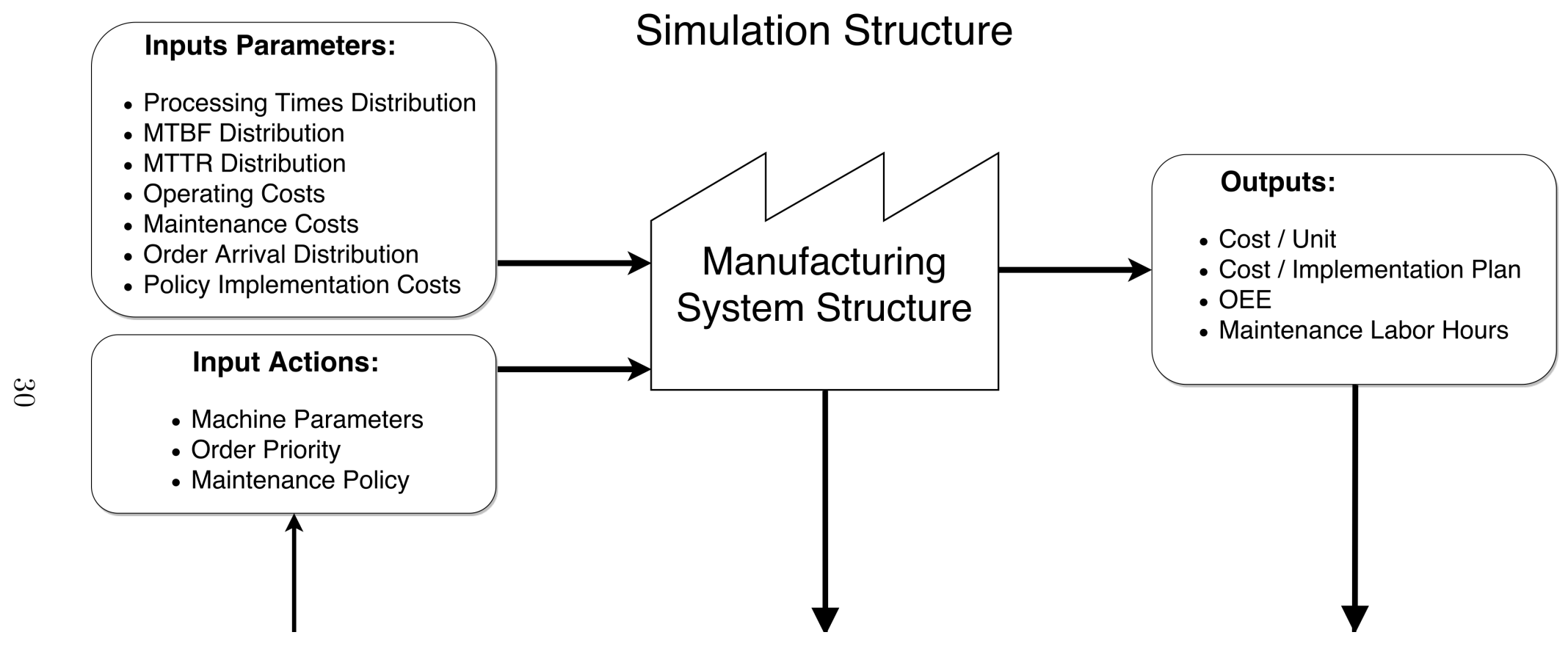

Figure 6: This a representative view of the simulation structure where parameters such as processing times and mean time between failure $(\mathrm{MTBF})$ are treated as inputs. The inclusion of machine parameters is a new consideration that will allow for the analysis of decision strategies that employ PHM information. 
equipment in the manufacturing process, distribution for each component's mean time between failure (MTBF), distribution for each component's mean time to repair (MTTR), operating costs, maintenance costs, equipment operating parameters, order schedule, and policy implementation costs. The outputs represent data needed for evaluation and presenting the business case. These include cost per unit produced, cost of implementing a particular maintenance policy, overall equipment effectiveness, and maintenance labor hours. The choice of these metrics is explained further in the next stage. The manufacturing system structure is the environment representing the unique arrangement of a company's hierarchical production facility, and will be driven by the work completed in step three. Most software packages on the market today, including MATLAB which is used for demonstration in Chapter 4, will be capable of building a simulation in this manner.

Step seven is to make pilot runs and check if the simulation is producing valid and useful results. In reality, the simulation should be tested at every step of the build process. Quality checks early on in the building step will prevent large and potentially project delaying changes later. If the real manufacturing system is at your disposal, checking your simulation with real data operating under the current maintenance policy is a great way to identify errors.

Step eight and nine are to design simulation experiments and make production runs, respectively. Much literature is available with methods of creating scenarios and measuring the impact. Generally speaking, the length of each simulation run, use of a warm-up period, and number of independent simulation runs should be specified. For the PHM simulation method presented here, this step will start by running the simulation for each maintenance policy in the selected set developed in step two. The 
first set of experiments will be to collect output data for the costs and equipment utilization metrics by keeping all manufacturing system inputs the same and only varying the maintenance policy input. Further experiments should be discussed with the project team and conducted to bolster the business case presented to decision makers. The length of production runs made may depend on the complexity of the model and computing power available to the systems analyst. From a manufacturing standpoint, a unique consideration would be to ensure an entire product cycle is simulated. For example, a manufacturer might have seasonality in the SKU's produced and varying types of changeovers necessary at different times of the year (or longer depending on product being made). In order to fully understand the impacts of a particular maintenance policy, the entire production schedule cycle should be simulated. Steps eight and nine should be repeated as new information becomes available or different experiments are designed.

\subsubsection{Metrics, Analysis, and Trade-offs}

With the production runs complete, data needs to be collected for the unique metrics of this thesis's simulation method covered in step ten. If the simulation is built according to the structure presented in step six, the output data should already be available and ready for analysis. The output metrics were selected for their ability to present a sound business case for decision makers and were validated with help from industry visits after speaking with manufacturing experts. Overall Equipment Effectiveness (OEE) is defined as:

$$
\mathrm{OEE}=A(t) * P(t) * Q(t)
$$

Where, 


$$
\begin{aligned}
& A(t)=\text { Availability }=\frac{\text { Scheduled Time }- \text { Downtime }}{\text { Scheduled Time }} \\
& P(t)=\text { Throughput }=\frac{\text { Units Made } * \text { Theoretical Cycle Time }}{\text { Scheduled Time }} \\
& Q(t)=\text { Quality }=\frac{\text { Good Units }}{\text { Total Units }}
\end{aligned}
$$

This is a measure well known to advanced manufacturer's using Lean Manufacturing tools such Total Productive Maintenance (TPM), and is considered an essential measure of ensuring low waste[37]. World class OEE is considered to be any organization that can achieve above $85 \%$ for their production facility. World class facilities that have achieved that number are famously operated by several automakers including Toyota Motor Corporation.

Cost per unit, or sometimes simply called unit cost, is calculated by adding up total production costs and dividing by the number of units produced. This is an important departure from merely considering total production costs, and is a widely used measure across industries. The cost variables to be modeled and accumulating as simulation time progresses are [38]: 
$C_{u}=$ Production cost per unit

$C_{c}=$ Replacement cost for a failed component

$L_{o}=$ Labor cost for operations employees

$L_{m}=$ Labor cost for maintenance employees

$S_{m}=$ Maintenance supplies cost

$M_{s}=$ Material cost, used to under cost of scrap

$H_{u}=$ Overhead cost for utilities

$R_{s}=$ Lost revenue due to stock outs, e.g. sales

$T_{s}=$ Transport cost, and other supply chain concerns

The cost of implementing a maintenance policy is a measure that will help for return on investment (ROI) calculations and be presented to the decision makers in a trade-off analysis in the next step. This is the single biggest driver of whether plant managers will choose to implement PHM into their facility. Cost of implementing specific policies needs to be estimated for direct comparison. Through working with our industrial manufacturing partner, costs could be incurred from but are not limited to:

- Maintenance tracking and reporting software package purchase, subscription fees

- Sensors purchase and installation

- Sensor activation fees (this occurs when physical sensors are already installed 
but equipment vendors charge to "activate" data collection and usability)

- Labor cost for employee to maintain, develop, implement, analyze, and update the maintenance system (the position in a company that handle this role may include a Reliability Engineer, Manufacturing Engineer, Maintenance Planner, Maintenance Coordinator, etc.)

- Data storage solution

- Time needed to train operators, mechanics, and management how to perform tasks required of a particular maintenance policy

Lastly, a measure that was consistently demanded through industry visits as is also a topic of some current literature is the number of maintenance hours needed for each particular policy [39]. The reason this is an important metric is due to two reasons (1) the average age of a maintenance employee, including both mechanics and electricians, is rising steadily for advanced manufacturers and (2) corporations are having trouble hiring new maintenance personnel and keeping turnover rates low. This has become such a common problem that companies will now often incentivize employees to join their organization with higher pay and funding education programs.

Steps eleven and twelve are to analyze trade-offs and to document and use results, respectively. The trade-offs analyzed depend on the experiments designed in step eight. The systems analyst should present trade-off curves for the purpose of showing how much unit cost and OEE are impacted between different policies. Plotting these standard charts will provide valuable insights for the company:

- Unit Cost versus OEE 
- Unit Cost versus Cost to Implement Policy

- OEE versus Cost to Implement Policy

- Number of Maintenance Hours versus Cost to Implement Policy

Additional experiments are welcome by varying input parameters of each maintenance policy. These can be used to further augment the business case and help decision makers feel more comfortable with a holistic view of the system. An example of this would be an experiment designed to answer the question: what would the MTTR have to be before preventive maintenance activities are not worth the cost? Of course, after trade-off analysis work is complete it should be documented and presented in a clear and concise manner to manufacturing leadership. The decision makers should be informed of the model building and validation processes, pointing-out assumptions where they exist. The decisions should be made based on the analysis provided there is agreement on model validity. During this step, adding simulation animation to the manufacturing system could help convey the message.

Step thirteen is to update data and policies when new information becomes available or new policies are developed. This is a step that could be automated and potentially tied-in directly to data systems already in place. Information being gathered on the production floor by the programmable logic controllers (PLC's) has the ability to be collected and auto-fitted to obtain distribution parameters which then can be fed into the PHM simulation method. Outputs could also be auto-generated and systems analysts alerted to new developments on a regular basis. It will be most important to update data and review new results if product demand climbs or significant process improvement were recently made. 


\subsection{Simulation Design}

The simulation architecture employs common design components and organization found in discrete event simulations where the next event will advance the simulated time [4]. These components are:

- Simulation clock: Maintains the currents simulation time as a state variable

- System state description: Variables used to describe system state at a specific time

- Statistical counters: Variables used to collect metrics on the system

- System entities: Items of interest that makeup the simulation

- Events: An action or occurrence that changes the state of the system

- Events list: A list populated with times of scheduled events, updated by the simulation program

- Simulation program: The main program that coordinates various subroutines to determine the schedule of events, update system state variables, terminate the simulation, and control reporting

The architecture of a PHM simulation for SMS must be adapted to include an expanded decision space for the ability to consider new policies. This can be seen in Table 1 with the addition of a state variables for machine parameters such as run speed or feed rates and machine health. 


\begin{tabular}{|l|l|}
\hline State Variable & $=\{$ Possible States $\}$ \\
\hline Machine Parameters & Run Speed, Feed Rates, etc. \\
\hline Machine Queue Length & $0,1,2,3, \ldots$ \\
\hline Machine Health & Good, Pre-Fail, Fail \\
\hline Machine Status & Busy, Idle, RM, PM \\
\hline Maintenance Times & $\geq 0$ \\
\hline Order Arrival Times & $\geq 0$
\end{tabular}

Entities in a PHM simulation design would include but are not necessarily limited to: manufacturer orders or products being made, machines, machine queues, material handlers, etc. Entities have attributes that can help track their state during all simulation time. The system state can only change by the occurrence of an event or action. In a PHM system, events and actions can be defined as the following:

- Machine Failure (MTBF)

- Maintenance Activities (MTTR)

- Machine Processing Activities

- Order Arrivals

- Change Parameters of Machine

- When to Perform Maintenance

- Change Maintenance Policy

Statistical Counters will be necessary to collect metrics for comparing the maintenance policies and ultimately performing trade-off analysis. The statistical variables 
used in a simulation for evaluating PHM policies should include but may not be limited to the following:

- Order Received, Orders Finished

- Total Maintenance Hours

- Number of Failures

- Average Length of Queue

- Orders Served by Machine

- Scrapped Parts

- PM Time

- RM Time

- Processing Times

Similar to a majority of discrete event simulations, the simulation logic functions as a combination of subroutines organized by the main program. The subroutines are for initialization, order arrival event, order departure events, machine failure event, changing machine parameter action, and a preventive maintenance event. These are depicted below with a description of the simulation algorithm and simplified diagram for each. 


\section{Initialization at $t_{0}$ :}

- Obtain and schedule the first order arrival time, $A_{1}$

- Obtain machine failure time

- Obtain machine processing time

- Initialize states and statistics, advance clock to the first event

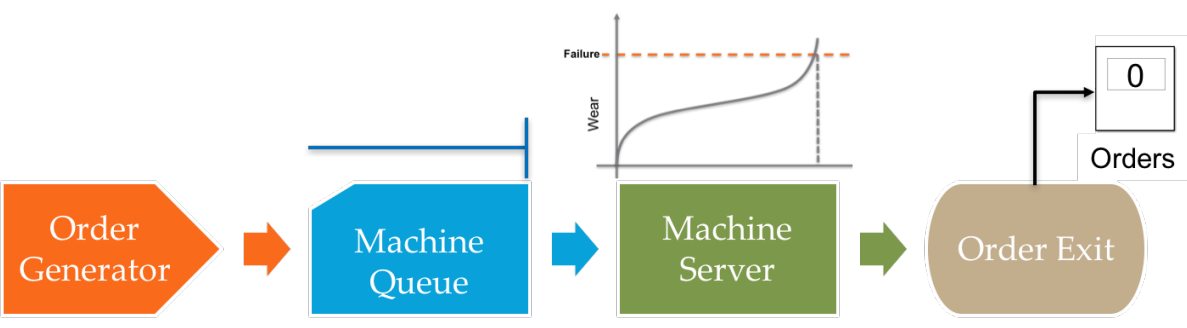

At simulation initialization, no orders are in the system therefore queue length is 0 , machine parameters are set to their default, machine health is good, and machine status is idle. No orders have entered or exited the system, therefore statistics are all still at initial values.

\section{Order Arrival at $t_{i}$ :}

- Next order time $A_{2}$ is scheduled in events list, the first order departure time $D_{1}$ scheduled

- If the machine is idle, the order enters and changes machine status to busy

- If machine status was busy, the order goes to machine's queue 
- Maintenance subroutine updates health states and will schedule maintenance activities with respect to policy

- In a corrective policy, $F_{t}$ scheduled if $F_{t}<D_{t}$ else departure occurs as planned

- In either a TBM and CBM policy, a preventive maintenance time $P M_{t}$ is scheduled if $P M_{t}<F_{t}<D_{t}$

- All states and statistics are updated with new values where applicable. Advance clock to the next event.
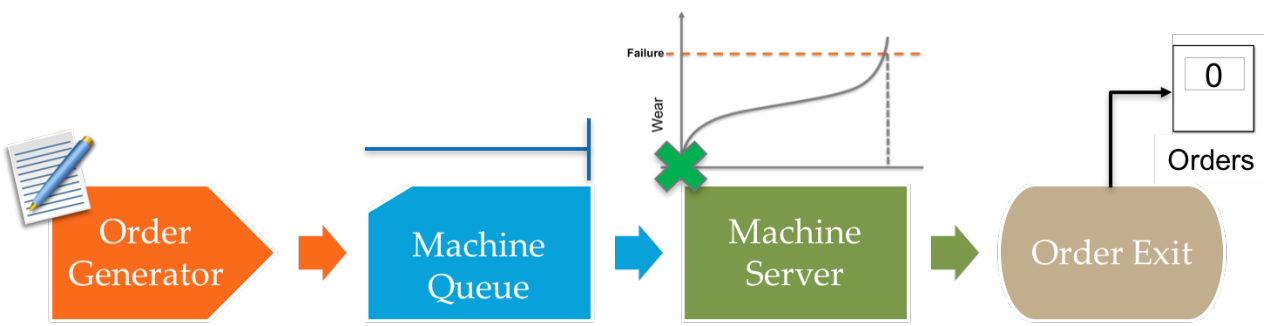

At this order arrival, because the simulation checked to see if the machine was busy and found it idle, the order will pass through the queue and enter the machine. This will change the machine status to busy. No machine parameters have been changed, queue length remains at 0 , and machine health is still good.

\section{Order Departure at $t_{i}$ :}

- If parts are in queue, let first one flow into the machine

- If the machine queue is empty, change the machine status to idle

- Maintenance subroutine runs to update machine health 
- All states and statistics are updated with new values where applicable. Advance clock to the next event.

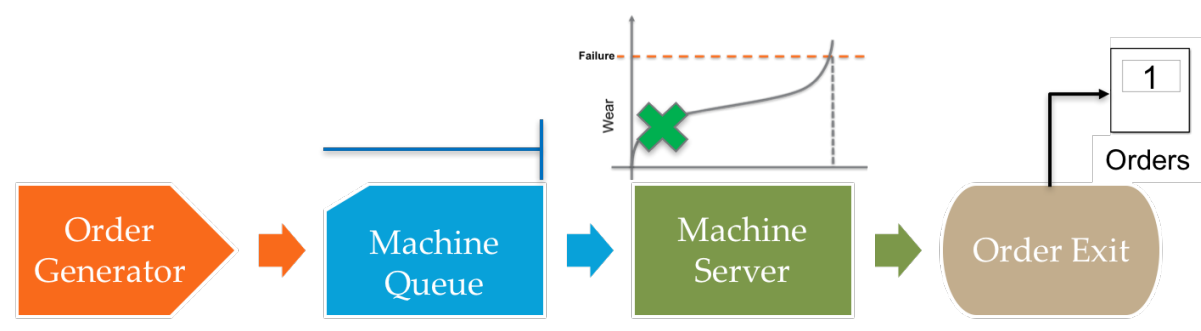

After this order departure, when the subroutine checked the machine queue, no more orders were found. Therefore the machine status was updated to idle. The machine health was checked and reported to still be good. No machine parameters were changed and queue length remains at 0 . Statistics were updated, and as an example, the orders completed statistic was updated to 1 in the above graphic.

\section{Machine Failure at $t_{i}$ :}

- At the last event, the maintenance subroutine checked health state and scheduled failure $F_{i}$

- If the failure time is scheduled to occur before a preventive maintenance event, then a failure event will occur and a corrective maintenance event will be scheduled

- When $C M_{t}$ time ends, the program obtains a new failure time from the inputted failure distribution and updates the health state

- Update arrival, departures event times to reflect maintenance delay 
- All states and statistics are updated with new values where applicable. Advance clock to the next event.

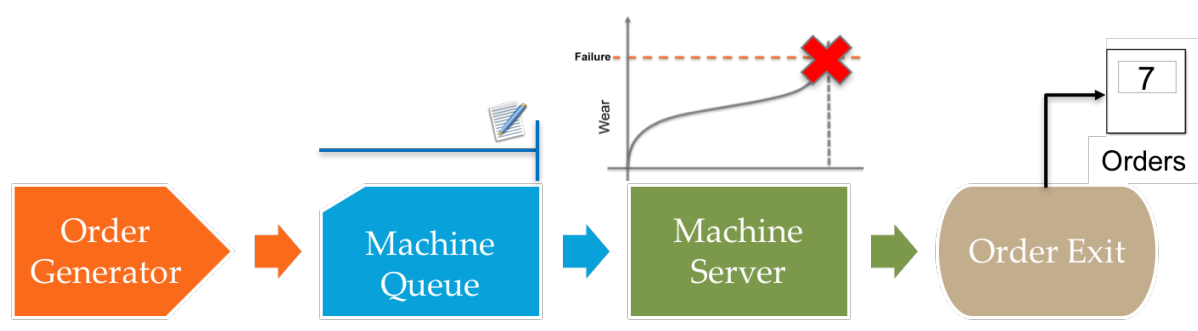

After a machine failure, the machine status will change to reflect a corrective maintenance event. Machine health state will be updated dependent upon effectiveness of the maintenance procedure. Queue length and machine parameters will also be checked along with statistics. As an example, shown above there is a queue length of 1 and orders completed at 7 .

\section{Machine Parameter Change at $t_{i}$ :}

- Based on machine health or other factors, an action is scheduled next in the events list to change a machine parameter e.g. speed to a new setting (often with the goal of maximizing uptime between failures)

- Maintenance subroutine runs to determine necessary update to $P M_{t}$ time if, for example, scheduling a preventive maintenance event after finished processing order $j$

- Since machine parameters have changed, update with a new failure time $F_{t}$ from new wear curve 
- Update arrival, departures event times to reflect new machine parameters

- All states and statistics are updated with new values where applicable. Advance clock to the next event.

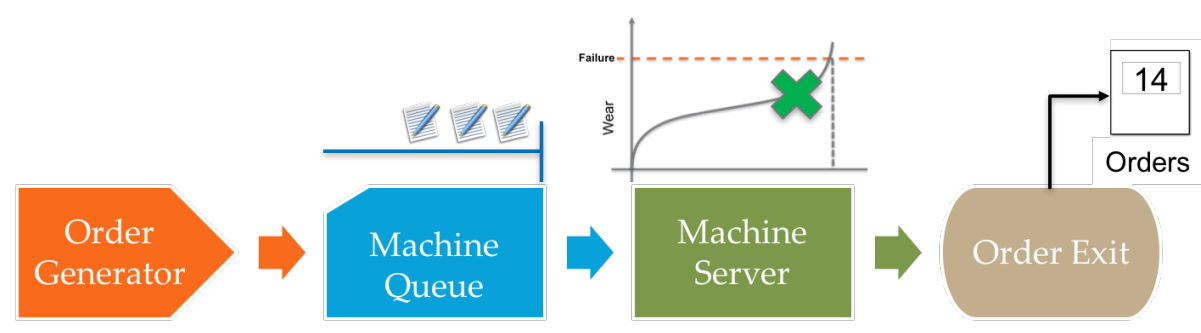

A machine parameter, such as speed rate, could be advantageous to change for prolonging the life of an asset. After a parameter is changed in the simulation, the corresponding parameter state change occurs. Machine health is updated to reflect any change in the wear curve. As an example, shown above there is a queue length of 3 and orders completed at 14 .

\section{Preventive Maintenance Performed at $t_{i}$ :}

- After the last event, the machine health state is still reported as prior to failure

- In the events list, $P M_{t}<F_{t}$ thus $P M_{t}$ will occur as scheduled

- Update health state to good, report out, receive action to schedule machine speed to high

- Update arrival, departures event times to account for delay from PM

- All states and statistics are updated with new values where applicable. Advance clock to the next event. 


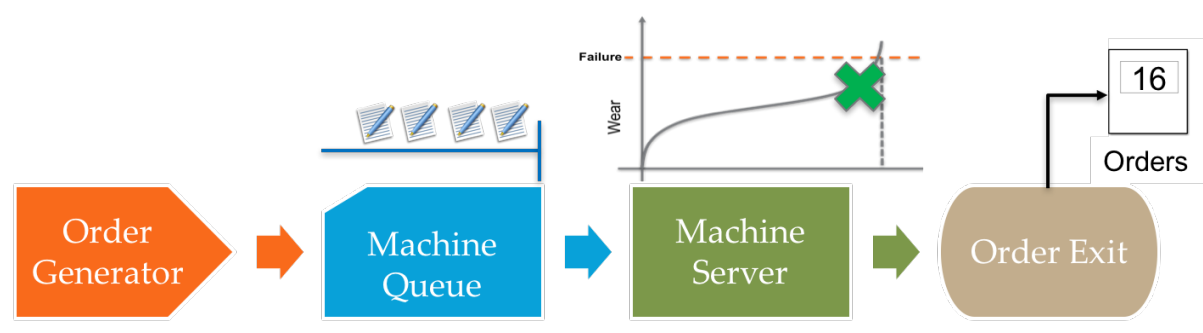

For this type of event, the machine status will update to reflect it is in preventive maintenance. After the PM has been performed, machine health will be updated dependent upon efficiency of maintenance completed. In the final example above, orders completed are at 16 and the queue length has grown to 4 .

\subsection{Chapter Summary}

This chapter gave a detailed description the PHM simulation methodology for SMS. The simulation methodology is specific to PHM in smart manufacturing through the addition or adaptation of steps one, two, three, six, ten, eleven, and thirteen. This can be seen from a comparison of Figures 3 and 5. It also discussed simulation's design including architecture and algorithm. The chapter provided the necessary setup for the discussion in Chapter 4 where the method is demonstrated for an advanced manufacturer. The demonstration will also serve as a validation of the method. 


\section{Demonstration of Method}

\subsection{Chapter Overview}

This chapter reviews a demonstration of the PHM simulation method introduced in

Chapter 3. It is demonstrated with the help of an actual industry partner, kind enough to open its doors for data collection and expert interviews for an extended period of time. The company represents an advanced manufacturing environment, but for the purpose of confidentiality will remain nameless in this thesis.

\subsection{Advanced Manufacturer Implementation}

The company represents an advanced manufacturer setting, operating a smart manufacturing system, and is interested in reviewing the business case for implementing PHM policies. The company specifically noted that they sought to:

1. Improve their low OEE performance 
2. Combat an inability to hire more maintenance workers by reducing the needed number of maintenance labor hours

3. Continually decrease unit cost to remain competitive

Therefore, the company is a great candidate for demonstration of the PHM simulation methodology for SMS.

Following the implementation program represented in Figure 1 and the simulation methodology, the team started by applying the risk analysis techniques of HHM, RFRM, and FCA to identify prioritized targets for their PHM system. This was done with the addition of PHM-specific rules for filtering through scenarios. At the equipment level, the cutoff saw, lathes, and five-axis machines were identified to be the prioritized targets for PHM implementation.

The manufacturer warranted the selection of the following set of policies to be evaluated:

- Corrective Maintenance (CM) - Maintenance activities are only performed after a failure has occurred. Here it is assumed the component will be replaced after the first failure.

- Time-Based Maintenance (TBM) - Maintenance activities are performed when a component reaches a predetermined time $T$ or fails.

- Conditioned-Based Maintenance (CBM) - The decision to perform maintenance is reached by observing a condition of the system and its components. It will be assumed the health state can be determined, and thus, the condition to perform maintenance is set at a pre-failure health state. 


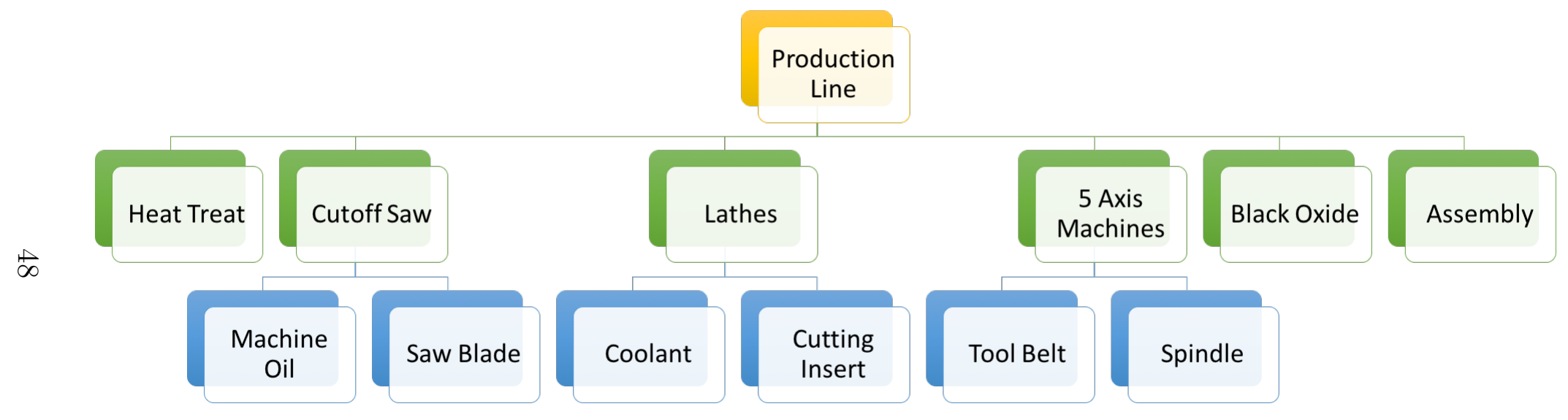

Figure 7: The manufacturer's production line hierarchy. These are purposely listed as general machines and components to protect the manufacturer's privacy. 
This is owed to finding their current maintenance policy to be a hybrid of corrective and time based maintenance; most components, however, were corrective. Opportunity was especially noticeable for the components prioritized from step one, which were largely operating on a corrective policy. Therefore, it was decided a comparison of the three main categories of maintenance policies would prove to be beneficial. This would allow the company to understand a broad spectrum of maintenance impacts on cost and OEE metrics. CBM in particular would represent the future of the company's factory if it were to use PHM methods. The company had some limited abilities to collect and analyze data in a diagnostic and prognostic manner, with the biggest obstacle being limited knowledge and training.

The structure of the smart manufacturing system was formed as illustrated in Figure 7. It is hierarchical in nature to ensure capturing the necessary complexity of the manufacturing system, but only for the resolution necessary to evaluate cost and OEE impact. Step one used risk analysis techniques to inform the decisions made on the level of detail required. A particular production-line was decided to be the highest level, as it represented the greatest opportunity for the plant and was similar to the other manufacturing lines. Thus, results from the chose production line could be generalized to the rest of the factory network. The risk filtering performed in step also informed the remaining levels of the operations hierarchy. Certain equipment and their respective components were omitted at the middle and lowest levels.

Data was collected from a variety of sources, including a maintenance information system, enterprise resource planning (ERP) system, and expert interviews using a standard list of questions. The company had been collecting maintenance data on MTBF, MTTR, and replacement costs for over a year. They made the entirety of 
that system available for the project team to review. The data was primarily best fit using a Weibull distribution for both failure and processing times. This is a well known and widely used distribution in the reliability literature, as discussed earlier. An example of the data being fit for lathes processing times is shown in Figure 8.

The assumptions made for the simulation included:

- Not incorporating material handling simulation, as this represented a process which took up practically zero time and never failed. Parts were delivered primarily by hand to the next station

- The order schedule was built from the company's most frequently demanded and produced SKU's

- The black oxide, heat treat, and assembly processes were only modeled to represent their variation in process times. There was not enough data to support failure modeling and they are not bottle necks in the operation

- When a component fails it will not be replaced according to a grouping approach

- Maintenance work will be a highly efficient operation and therefore components will be considered as good as new (AGAN)

Next, the simulation was built following the input-system-output structure introduced in Chapter 3. Figure 9 is a screen shot of the MATLAB simulation environment for the Tool Company's production line.

MATLAB, and more specifically, the SimEvents Toolbox was used as the computerized simulation environment for programming the structure, inputs module, and 


\section{Weibull for Lathe Processing Times}

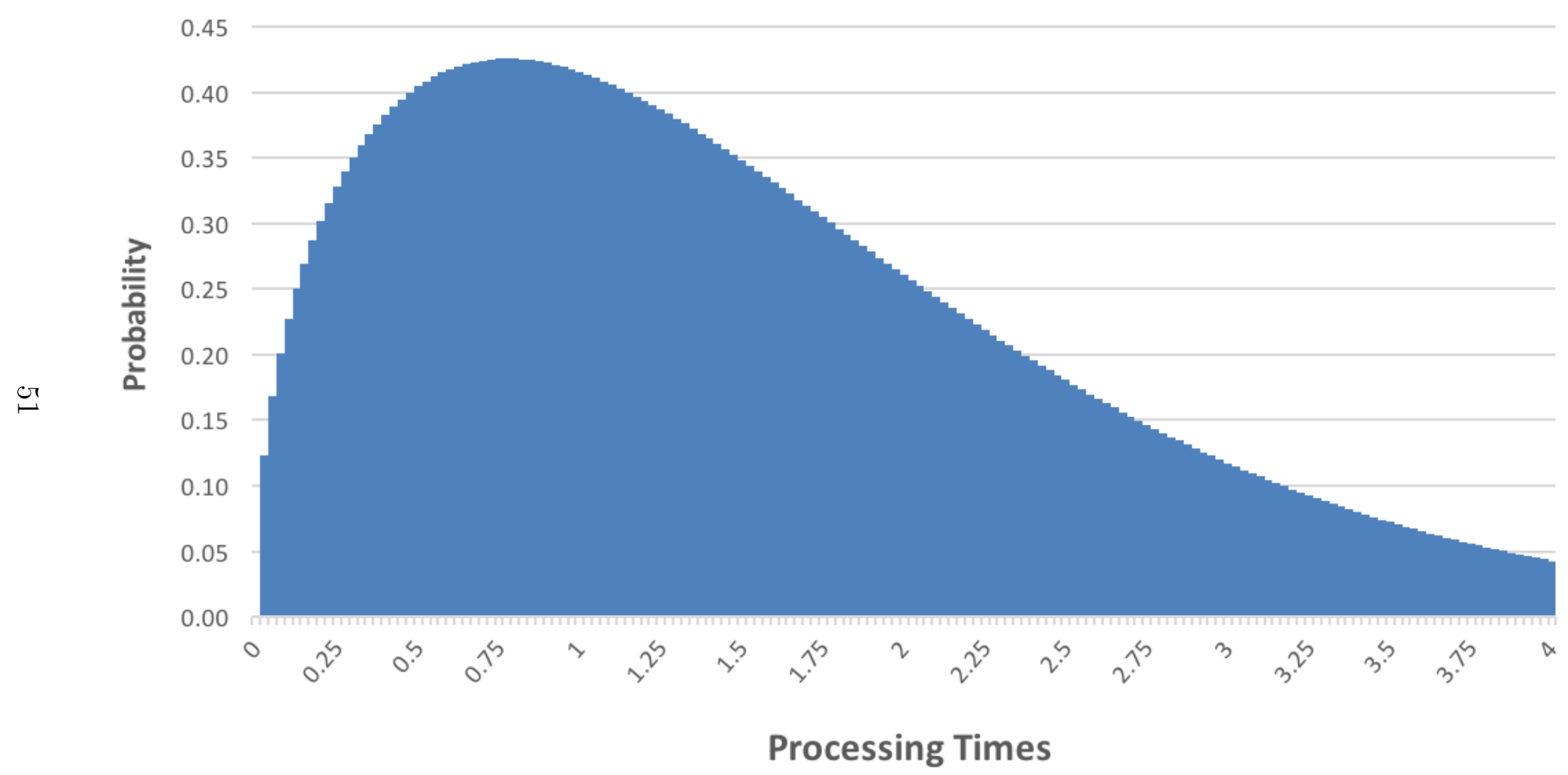

Figure 8: Lathe processing times fit to a Weibull distribution with shape parameter, $k=1.45$, and scale parameter, $\lambda=1.737$. 


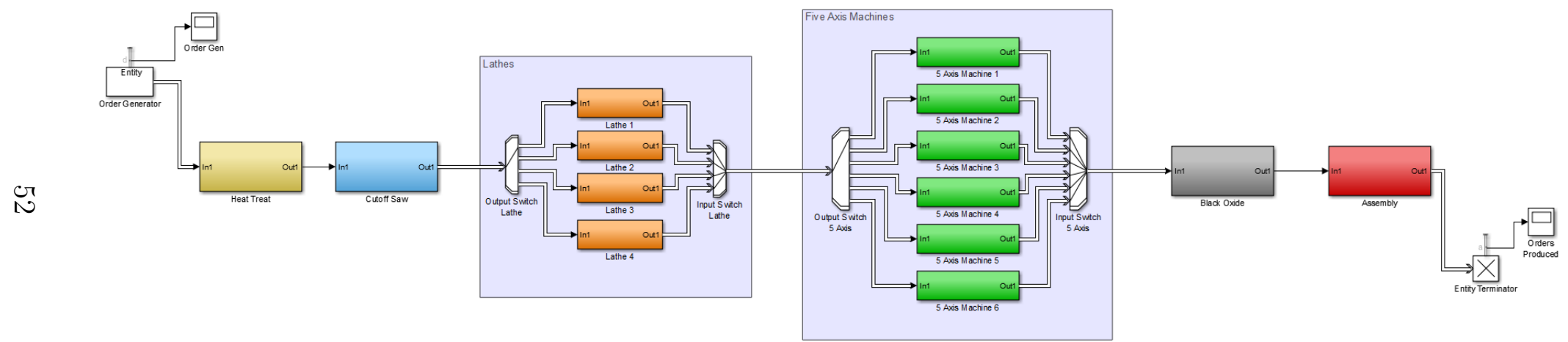

Figure 9: Simulation built in MATLAB for an advanced manufacturer implementation 
analysis reports. The simulation clock advances by the next-event time advance approach as discussed in [4], since all major simulation software packages work in this manner including MATLAB's SimEvents toolbox.

\subsection{Discussion of Results}

The results were documented and can be seen in the following figures. Figure 10 shows that mean cost per unit decreases and mean OEE rises as the Tool Company would head down the path towards implementing a condition-based policy. Error bars based on a $90 \%$ confidence interval are also included for reference.

Figure 11 details an important trade-off when considering the cost of implementing a particular policy versus gains in mean OEE. While the mean OEE does improve as the company moves towards a CBM strategy using PHM methods, it will cost significantly more capital to achieve less gains in OEE when moving from TBM to CBM as compared to Corrective to TBM. It will be up to the decision-maker as to whether the invested capital would be worth the return in OEE. Additionally, after examination through the use of a $90 \%$ confidence interval there is not strong evidence the policies are different since the intervals overlap. The decision maker would most likely conclude, based on this overlap and increased capital cost, to choose TBM versus CBM.

Figure 12 compares mean unit cost versus cost to implement a particular policy. This replicates a similar trade-off seen in Figure 11, and will again be up to the decision-maker is the margin expansion will be great enough to invest capital. For

example, the manufacturer might be planning to make significant product changes in the next couple years and thus would not be able to recoup their return on invest- 


\section{Cost per Unit vs. OEE}

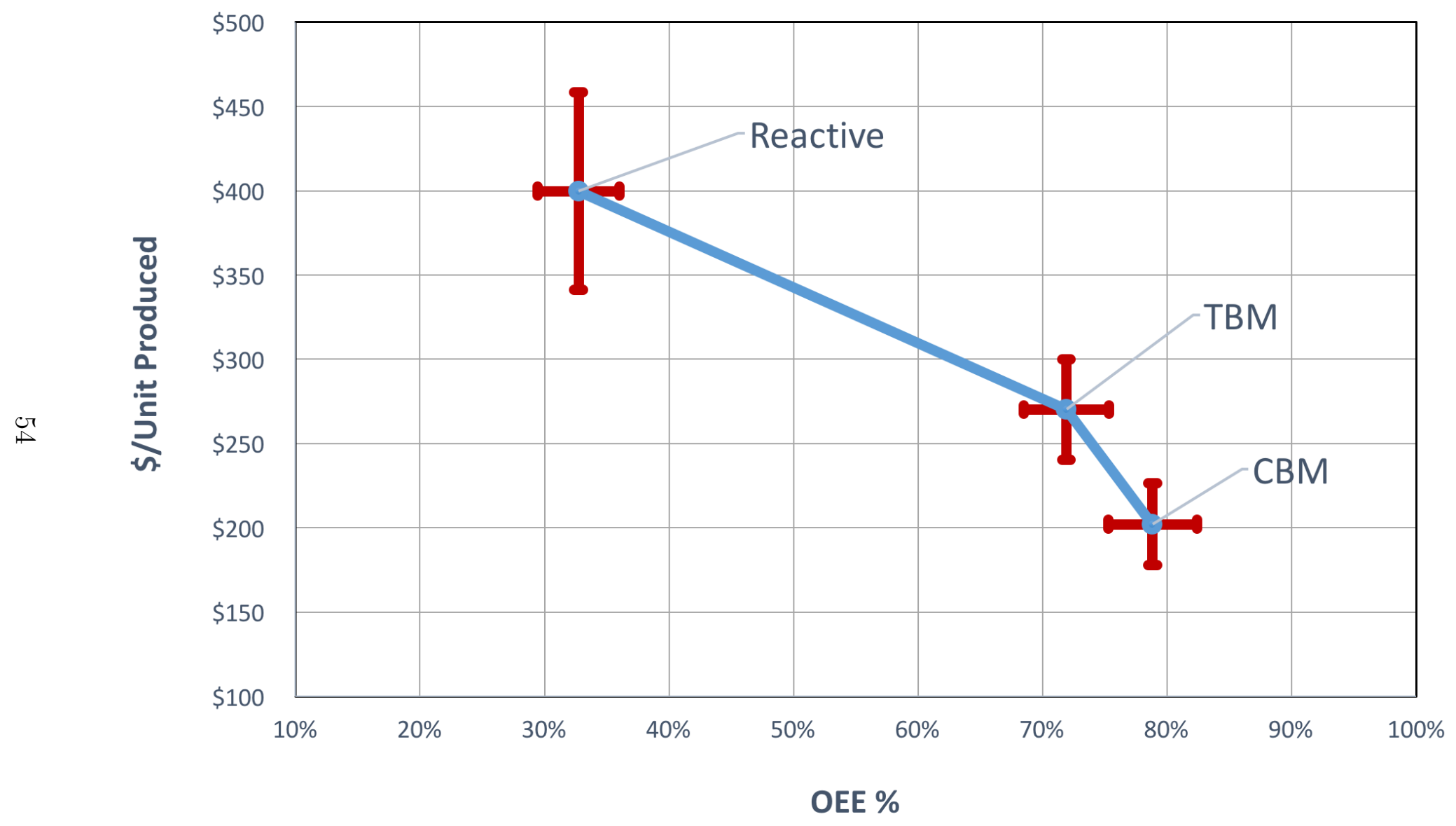

Figure 10: These results show the mean cost per unit decreases and mean OEE rises as the manufacturer drives towards implementing a CBM policy. Error bars for a $90 \%$ confidence interval are shown in red for each point. 


\section{Cost of Policy vs. OEE}

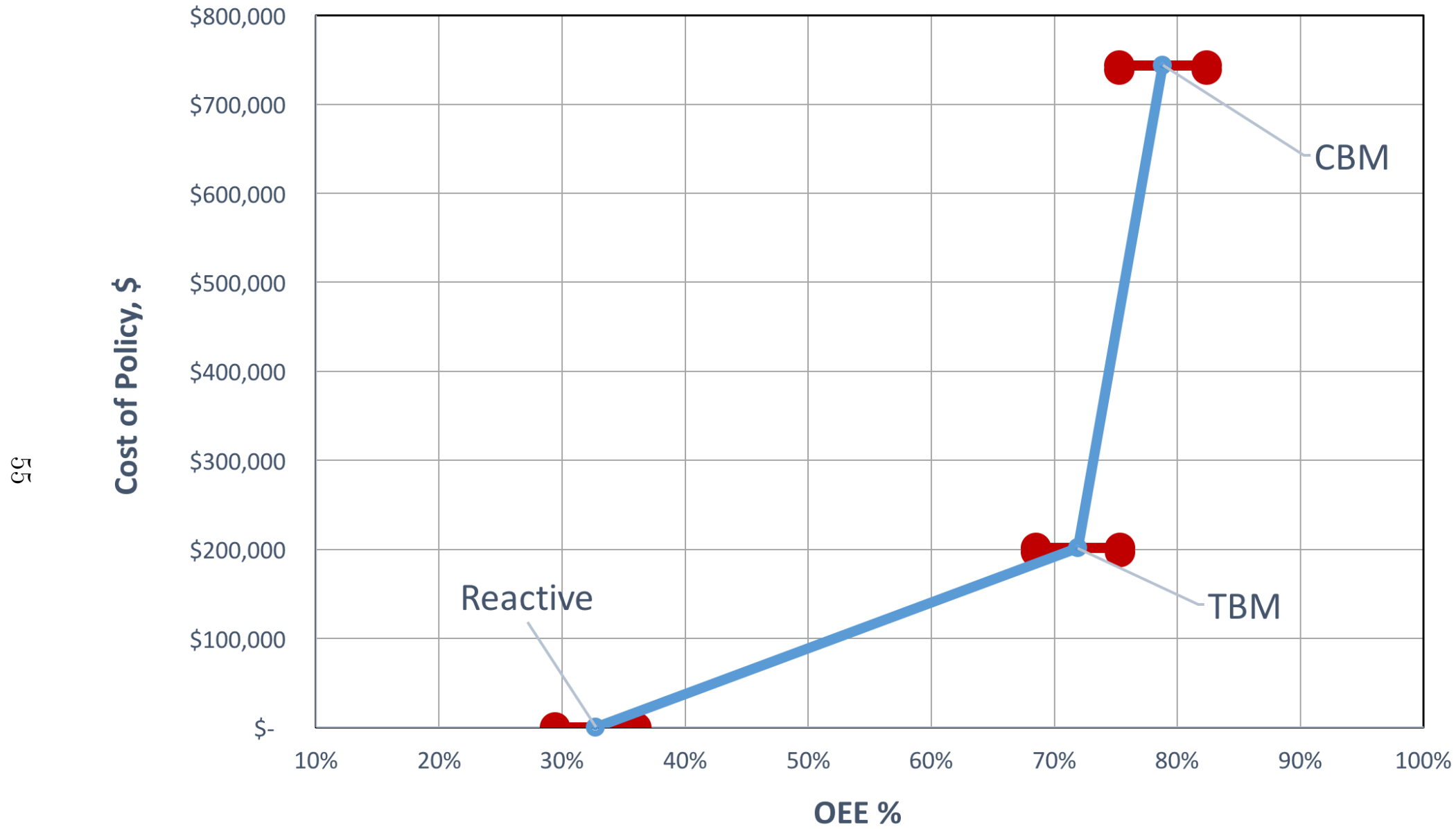

Figure 11: These results compare the cost to implement each policy versus mean OEE with error bars for a $90 \%$ confidence interval. There exists a trade-off where the cost to implement a CBM over a TBM policy results in a much smaller increase in OEE when compared to the gain in OEE realized from switching to a TBM policy from Reactive. 
ment. However, if the company expects they will maintain steady demand with little changes, any expansion of margin would be welcome.

Figure 13 represents the data which many maintenance managers and plant leadership have requested the project team to see: the impact on the number of maintenance employees required for a particular policy. As the three cumulative distribution function plots show, maintenance employees tend to decrease as PHM techniques are used. However, there is a bit of overlap between TBM and CBM. This might suggest that testing a hybrid policy where Reactive, TBM, and CBM methods are all deployed would produce smaller variations for required maintenance hours. Too much maintenance time is probably being spent on components that do not need it. 


\section{Unit Cost vs. Cost of Policy}

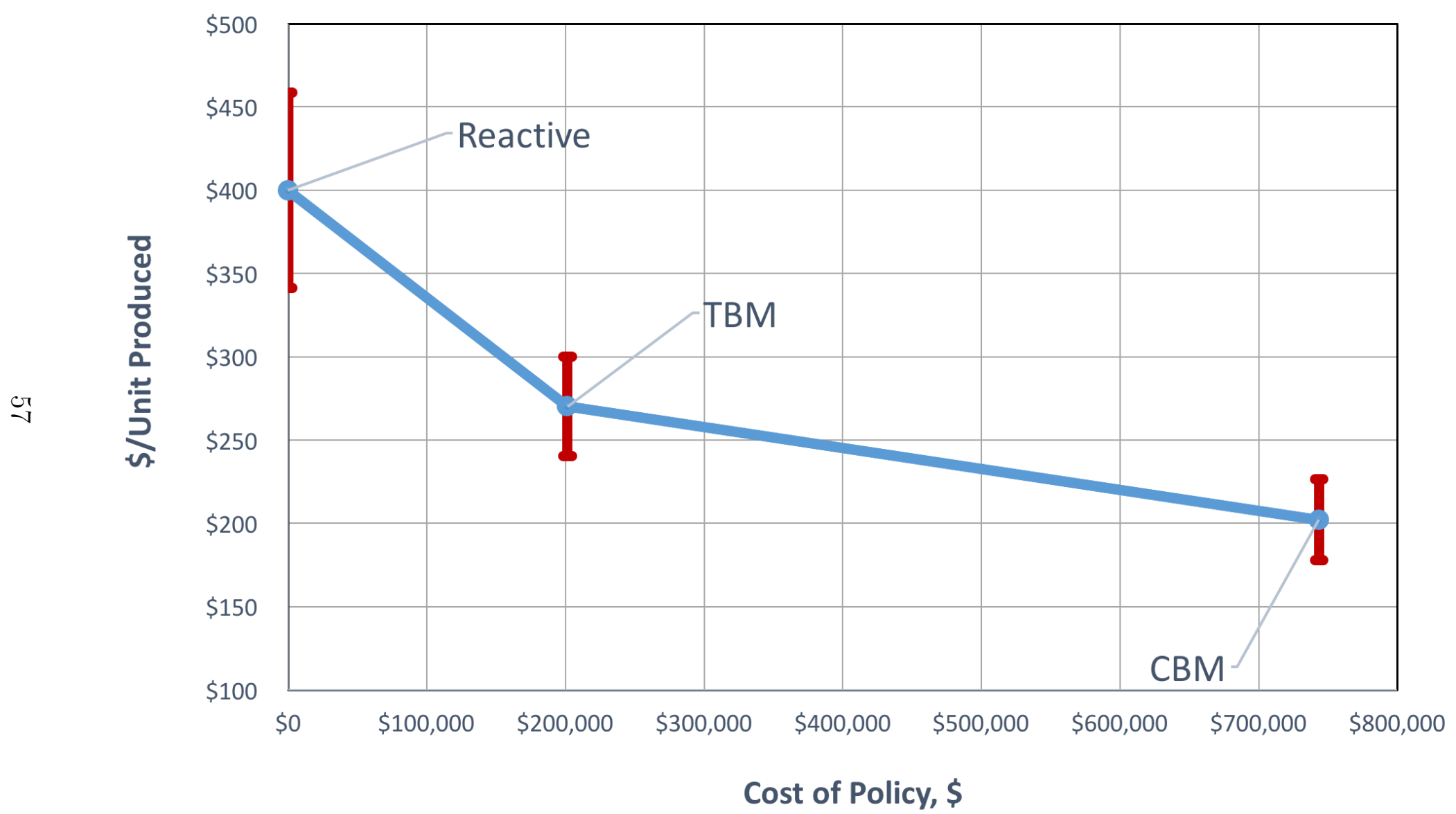

Figure 12: Mean unit cost with error bars for a $90 \%$ confidence interval are shown versus policy cost to implement. 
CDF, Employees Needed by Policy

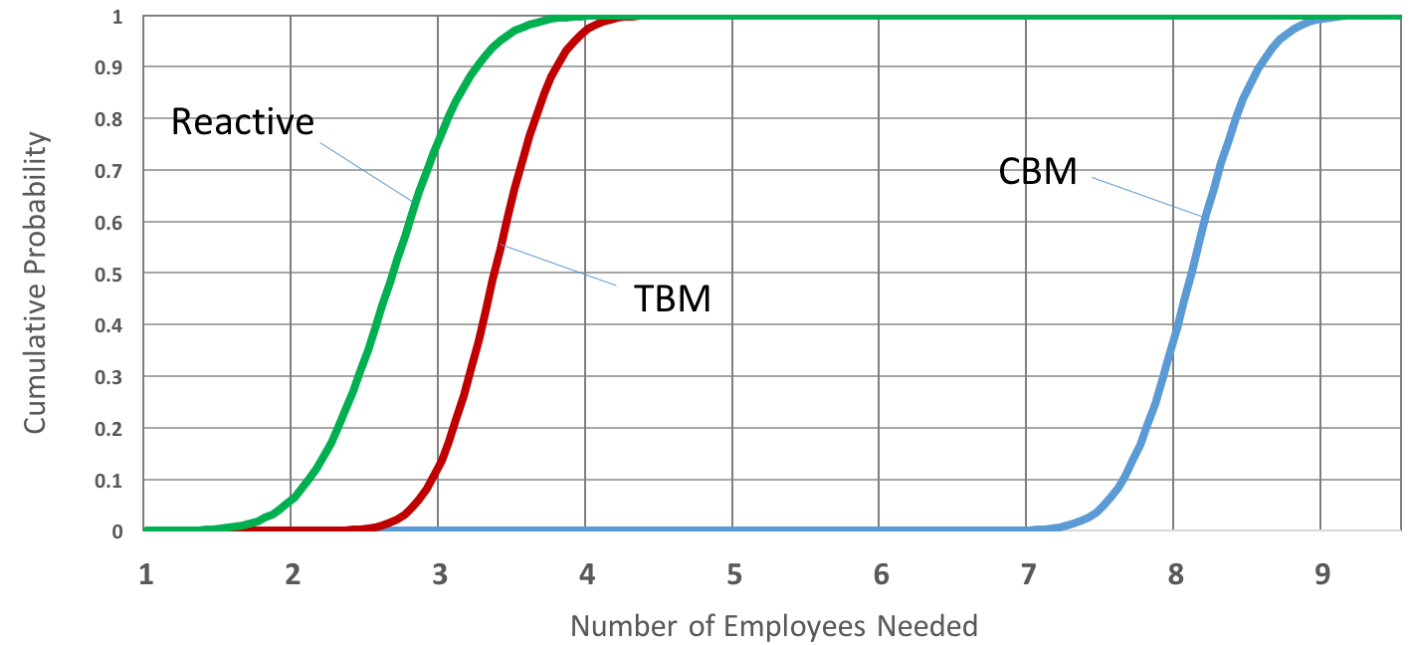

Figure 13: Maintenance labor hours were totaled and converted to show the number of employees needed for each policy as a Cumulative Distribution Function. 


\subsection{Chapter Summary}

This chapter demonstrated the journey of implementing the PHM simulation methodology for SMS. By doing so, the method was calibrated and validated to focus generalized simulation efforts with for study of PHM techniques in the advanced manufacturing industry. 


\section{Conclusions}

\subsection{Chapter Overview}

This chapter will discuss the major findings and contributions of the thesis. It will note observations from creating the simulation method and demonstration on an actual Tool Company manufacturing system. The contributions are expanded upon for the purpose of realizing where future work will be initiated. Figure 14 is introduced and discussed with respect to a possible model-free reinforcement learning application for optimization of maintenance and operations policies.

\subsection{Findings and Contributions}

Through deployment of the PHM simulation methodology for SMS, it was shown that manufacturer's can realize important trade-offs and valuable insights for their

business. It is an exciting realization of this thesis to recognize a great moment 
in time for advanced manufacturers to leverage their own data-rich systems. Many corporations are "sitting-on" mountains of data waiting to be exploited, and the PHM simulation method presented in this thesis is one such perfect application. An explanation for this might be that there is a lack of technical knowledge in many manufacturers' employees, especially at the production line level. The companies might not realize the benefit of budgeting for a data scientist or systems analyst; however, this use of the simulation method indicated otherwise.

Through the implementation of the simulation method at the advanced manufacturer, we found that Condition-Based policies do provide the biggest potential for cost savings. However, a caveat is that they should be implemented in areas where PHM will have the greatest impact. Components that take very short periods of time to replace with little or no impact on product quality during a failure would not be good candidates for condition monitoring. High-cost components that have a long mean time to repair will benefit the most from PHM. If data is not available for determining these components, interviews with production floor employees and other experts should quickly filter to the surface those high priority components. This was achieved in our own implementation through completing step one of the PHM simulation method with a combination of data and expert interviews.

A major contribution of this thesis is to provide advanced manufacturers a simulation method for investigation and preparation of a PHM business case for SMS. The field of simulation has supplied well established methods to build a general simulation study and rigorous frameworks for analyzing the relevance of its results. However, that field can be augmented and modified to fit the needs of manufacturers as they seek to implement a PHM policy in their facilities. 
Further major contributions of this work is the simulation structure, created for three benefits: first, to simulate novel PHM policies that extend the decision space to include controlling machine parameters, second, to add generality for the purpose of quickly updating input data to the model, and lastly, to provide the ability to integrate with a decision module for the purpose of determining optimal policies. This last contribution is illustrated in Figure 14.

\subsection{Future Work}

Much of the potential for future work revolves around expanding upon the contribution of the simulation's ability to integrate with a decision module. The decision module would be able to communicate with the simulation structure by sending actions to update input parameters. The manufacturing system processes those inputs and outputs are created; the manufacturing system will communicate state variables and the outputs report out rewards. A structure like this would be beneficial for the purpose of implementing a model-free reinforcement learning algorithm. The goal of such a reinforcement learning technique would be to build a maintenance and operations policy optimized to the component level.

Future work will also center around employing this method to build a simulation which can be updated with real-time data from the manufacturing process. This automation would present a way to reduce time spent gathering and fitting data. The simulation would simply be ready to produce output metrics and show their respective trade-offs immediately upon request. 


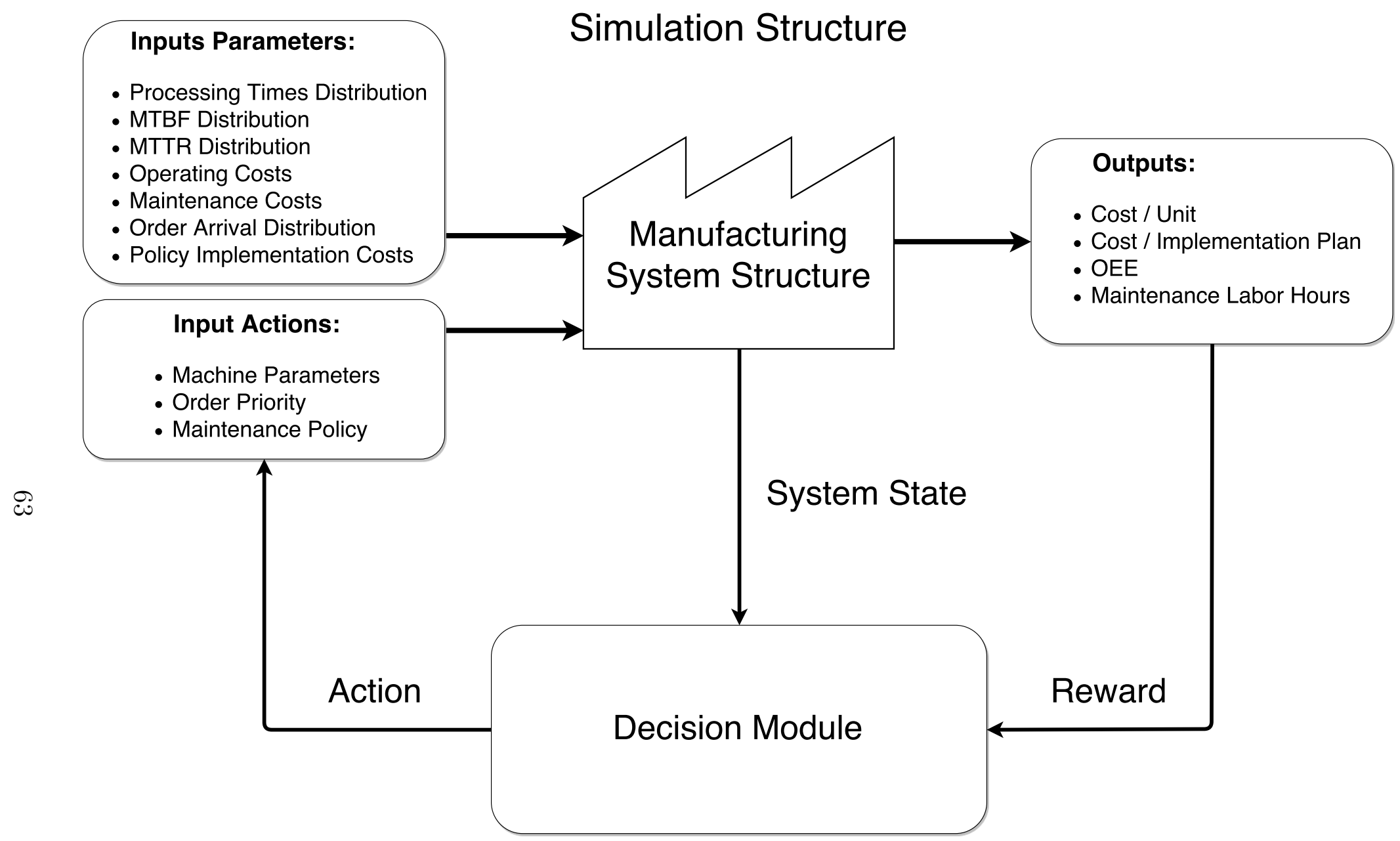

Figure 14: The simulation structure would allow for future work to be conducted by interfacing with a decision module, or controller, for the purpose of generating optimal operations policies by use of a model-free reinforcement learning method. The simulation could output both the state of the system and a reward to the controller where an action is produced to alter inputs parameters. 


\subsection{Chapter Summary}

This chapter concluded the work presented in this thesis by noting major contributions and findings. Potential topics of future work are introduced and indicate the on-going work happening at University of Virginia. It is certainly an exciting time for the field of advanced manufacturing. The data-rich environment is perfect for unlocking insights through data science techniques employed by PHM methods. The connectedness of smart manufacturing systems will also bring great advancements in providing decision support and automating our future. 


\section{References}

[1] J. Davis, T. Edgar, J. Porter, J. Bernaden, and M. Sarli, "Smart manufacturing, manufacturing intelligence and demand-dynamic performance," Computers $\&$ Chemical Engineering, vol. 47, pp. 145-156, 2012.

[2] A. Anderson, "Report to the president on ensuring american leadership in advanced manufacturing." Executive Office of the President, 2011.

[3] P. W. Kalgren, C. S. Byington, and M. J. Roemer, "Defining phm, a lexical evolution of maintenance and logistics," in Autotestcon, 2006 IEEE. IEEE, 2006, pp. 353-358.

[4] W. D. Kelton and A. M. Law, Simulation modeling and analysis. McGraw Hill Boston, 2000.

[5] P. A. Sandborn and C. Wilkinson, "A maintenance planning and business case development model for the application of prognostics and health management (phm) to electronic systems," Microelectronics Reliability, vol. 47, no. 12, pp. 1889-1901, 2007.

[6] E. Scanff, K. Feldman, S. Ghelam, P. Sandborn, M. Glade, and B. Foucher, "Life cycle cost impact of using prognostic health management (phm) for helicopter avionics," Microelectronics Reliability, vol. 47, no. 12, pp. 1857-1864, 2007.

[7] P. N. Muchiri, L. Pintelon, H. Martin, and P. Chemweno, "Modelling maintenance effects on manufacturing equipment performance: results from simulation analysis," International Journal of Production Research, vol. 52, no. 11, pp. 3287-3302, 2014. 
[8] B. S. Dhillon, Engineering maintenance: a modern approach. CRC Press, 2002.

[9] S. Duffuaa, M. Ben-Daya, K. Al-Sultan, and A. Andijani, "A generic conceptual simulation model for maintenance systems," Journal of Quality in Maintenance Engineering, vol. 7, no. 3, pp. 207-219, 2001.

[10] R. Barlow and L. Hunter, "Optimum preventive maintenance policies," Operations research, vol. 8, no. 1, pp. 90-100, 1960.

[11] J. J. McCall, "Maintenance policies for stochastically failing equipment: a survey," Management science, vol. 11, no. 5, pp. 493-524, 1965.

[12] I. Jeong, V. Leon, and J. Villalobos, "Integrated decision-support system for diagnosis, maintenance planning, and scheduling of manufacturing systems," International Journal of Production Research, vol. 45, no. 2, pp. 267-285, 2007.

[13] T. D. Batzel and D. C. Swanson, "Prognostic health management of aircraft power generators," Aerospace and Electronic Systems, IEEE Transactions on, vol. 45, no. 2, pp. 473-482, 2009.

[14] S. Holland, L. Barajas, M. Salman, and Y. Zhang, "Phm for automotive manufacturing \& vehicle applications," in Prognostics 83 Health Management Conference Fielded Systems Session, 2010.

[15] S. J. Hu and Y. Koren, "Stream-of-variation theory for automotive body assembly," CIRP Annals-Manufacturing Technology, vol. 46, no. 1, pp. 1-6, 1997. 
[16] T. Shen, F. Wan, W. Cui, and B. Son, "Application of prognostic and health management technology on aircraft fuel system," in Prognostics and Health Management Conference, 2010. PHM'10. IEEE, 2010, pp. 1-7.

[17] H. Wang, "A survey of maintenance policies of deteriorating systems," European journal of operational research, vol. 139, no. 3, pp. 469-489, 2002.

[18] I. B. Gertsbakh, Models of preventive maintenance. Elsevier Science \& Technology, 1977, vol. 23.

[19] Y. Peng, M. Dong, and M. J. Zuo, "Current status of machine prognostics in condition-based maintenance: a review," The International Journal of Advanced Manufacturing Technology, vol. 50, no. 1-4, pp. 297-313, 2010.

[20] R. Ahmad and S. Kamaruddin, "An overview of time-based and condition-based maintenance in industrial application," Computers $\mathscr{E}$ Industrial Engineering, vol. 63, no. 1, pp. 135-149, 2012.

[21] H. P. Bloch and F. K. Geitner, Machinery Failure Analysis and Troubleshooting: Practical Machinery Management for Process Plants. Butterworth-Heinemann, 2012.

[22] A. Van Horenbeek and L. Pintelon, "A dynamic predictive maintenance policy for complex multi-component systems," Reliability Engineering 83 System Safety, vol. 120, pp. 39-50, 2013.

[23] A. Gruber, S. Yanovski, and I. Ben-Gal, "Condition-based maintenance via simulation and a targeted bayesian network metamodel," Quality Engineering, vol. 25, no. 4, pp. 370-384, 2013. 
[24] B. Y. Choo, P. A. Beling, A. E. LaViers, J. A. Marvel, and B. A. Weiss, "Adaptive multi-scale phm for robotic assembly processes," in Annual Conference of the PHM Society (In review), 2015.

[25] M. Vineyard and J. Meredith, "Effect of maintenance policies on fms failures," The International Journal Of Production Research, vol. 30, no. 11, pp. 26472657, 1992.

[26] A. BANERJEE and B. Flynn, "A simulation study of some maintenance policies in a group technology shop," International Journal of Production Research, vol. 25, no. 11, pp. 1595-1609, 1987.

[27] R. Logendran and D. Talkington, "Analysis of cellular and functional manufacturing systems in the presence of machine breakdown," International Journal of Production Economics, vol. 53, no. 3, pp. 239-256, 1997.

[28] S. S. Padhi, S. M. Wagner, T. T. Niranjan, and V. Aggarwal, "A simulation-based methodology to analyse production line disruptions," International Journal of Production Research, vol. 51, no. 6, pp. 1885-1897, 2013.

[29] L. A. Huertas Quintero, A. A. West, D. M. S. Velandia, P. P. Conway, and A. Wilson, "Integrated simulation tool for quality support in the low-volume high-complexity electronics manufacturing domain," International Journal of Production Research, vol. 48, no. 1, pp. 45-68, 2010.

[30] A. P. Sage and Y. Y. Haimes, Risk modeling, assessment, and management. John Wiley \& Sons, 2015. 
[31] Y. Y. Haimes, "Systems-based guiding principles for risk modeling, planning, assessment, management, and communication," Risk analysis, vol. 32, no. 9, pp. 1451-1467, 2012.

[32] Y. Y. Haimes, S. Kaplan, and J. H. Lambert, "Risk filtering, ranking, and management framework using hierarchical holographic modeling," Risk Analysis, vol. 22, no. 2, pp. 383-397, 2002.

[33] M. Malinowski, P. Beling, Y. Haimes, A. LaViers, J. Marvel, and B. Weiss, "System interdependency modeling in the design of prognostic and health management systems in smart manufacturing," in 2015 Annual Conference of the PHM Society, 2015.

[34] R. E. Wildeman, R. Dekker, and A. Smit, "A dynamic policy for grouping maintenance activities," European Journal of Operational Research, vol. 99, no. 3, pp. $530-551,1997$.

[35] J.-K. Chan and L. Shaw, "Modeling repairable systems with failure rates that depend on age and maintenance," Reliability, IEEE Transactions on, vol. 42, no. 4, pp. 566-571, 1993.

[36] H. Pham and H. Wang, "Imperfect maintenance," European journal of operational research, vol. 94, no. 3, pp. 425-438, 1996.

[37] S. Nakajima, "Introduction to tpm: total productive maintenance," Productivity Press, Inc, P. O. Box 3007, Cambridge, Massachusetts 02140, USA, 1988. 129, 1988.

[38] W. J. Hopp and M. L. Spearman, Factory physics. Waveland Press, 2011. 
[39] D. W. DeLong, Lost knowledge: Confronting the threat of an aging workforce. Oxford University Press, 2004. 


\section{Appendix A: MATLAB Interface Subcomponents}

The following pages will show figures for the various subcomponents of the simulation. 


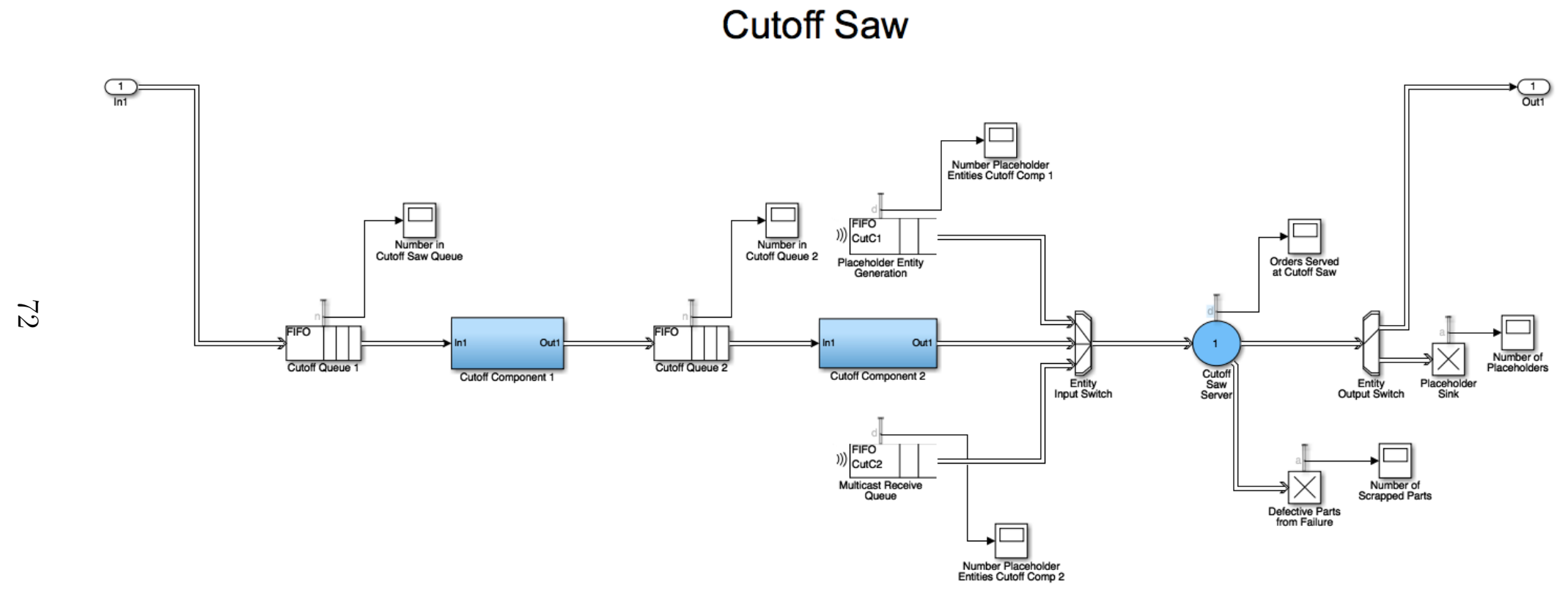

Figure 15: View of the cutoff saw process 


\section{Cutoff Saw - Saw Blade}

기

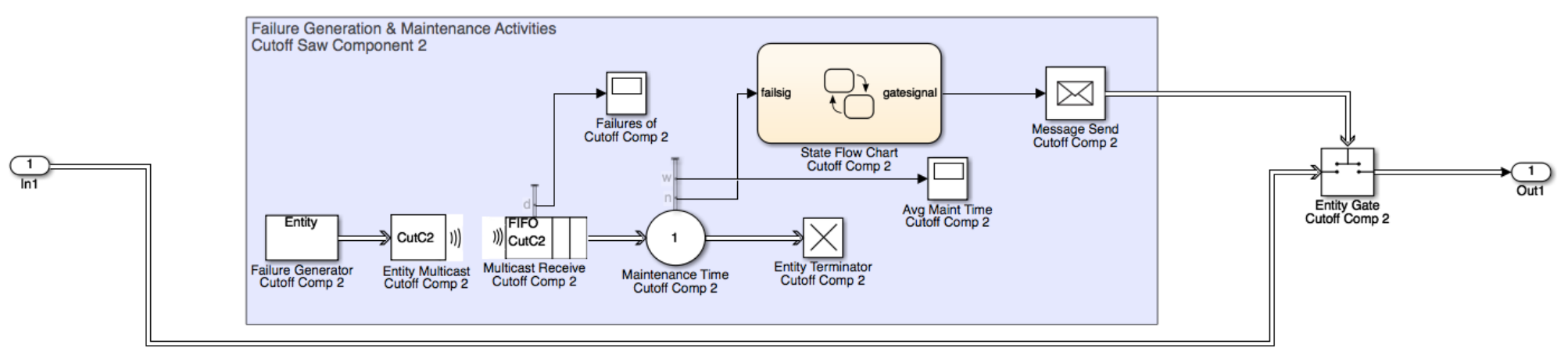

Figure 16: View of the process for a component of the cutoff saw, the saw blade. 


\section{Assembly Process}

I

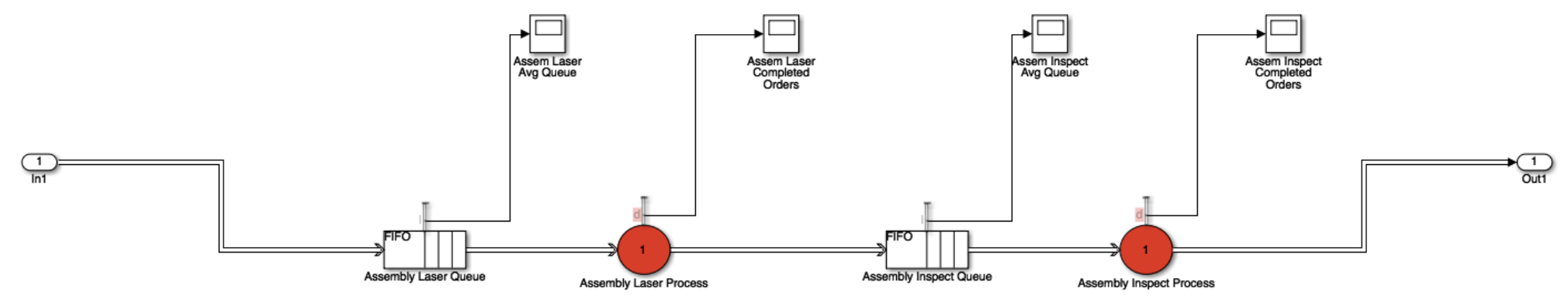

Figure 17: View of the assembly process, which has two servers for the laser and inspection processes, respectively. 
A Simulation Environment to Support Prognostics and Health Management in Smart Manufacturing Systems

\author{
A Thesis \\ Presented to \\ the faculty of the School of Engineering and Applied Science \\ University of Virginia \\ in partial fulfillment \\ of the requirements for the degree \\ Master of Science \\ by
}

Gerald R. Heddy, III

August

2016 
APPROVAL SHEET

The thesis

is submitted in partial fulfillment of the requirements

for the degree of

Master of Science

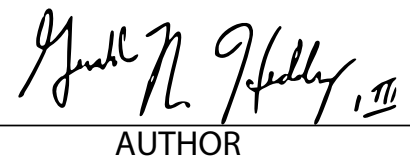

The thesis has been read and approved by the examining committee:

\begin{tabular}{c} 
Peter Beling \\
\hline $\begin{array}{c}\text { Advisor } \\
\text { Yacov Haimes }\end{array}$ \\
\hline Stephen Adams \\
\hline James Lambert \\
\hline Cody Fleming
\end{tabular}

Accepted for the School of Engineering and Applied Science:

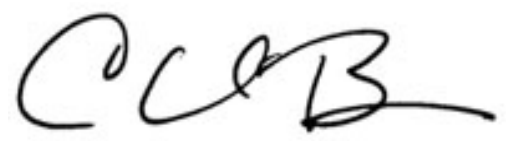

Craig H. Benson, Dean, School of Engineering and Applied Science

August 